\title{
Chemokines, costimulatory molecules and fusion proteins for the immunotherapy of solid tumors
}

In this article, the role of chemokines and costimulatory molecules in the immunotherapy of experimental murine solid tumors and immunotherapy used in ongoing clinical trials are presented. Chemokine networks regulate physiologic cell migration that may be disrupted to inhibit antitumor immune responses or coopted to promote tumor growth and metastasis in cancer. Recent studies highlight the potential use of chemokines in cancer immunotherapy to improve innate and adaptive cell interactions and to recruit immune effector cells into the tumor microenvironment. Another critical component of antitumor immune responses is antigen priming and activation of effector cells. Reciprocal expression and binding of costimulatory molecules and their ligands by antigen-presenting cells and naive lymphocytes ensures robust expansion, activity and survival of tumor-specific effector cells in vivo. Immunotherapy approaches using agonist antibodies or fusion proteins of immunomodulatory molecules significantly inhibit tumor growth and boost cell-mediated immunity. To localize immune stimulation to the tumor site, a series of fusion proteins consisting of a tumor-targeting monoclonal antibody directed against tumor necrosis and chemokines or costimulatory molecules were generated and tested in tumor-bearing mice. While several of these reagents were initially shown to have therapeutic value, combination therapies with methods to delete suppressor cells had the greatest effect on tumor growth. In conclusion, a key conclusion that has emerged from these studies is that successful immunotherapy will require both advanced methods of immunostimulation and the removal of immunosuppression in the host.

\section{KEYWORDS: chemokines costimulatory molecules fusion proteins immunotherapy regulatory T cells tumor microenvironment}

Despite recent advances, mainstream cancer therapies, namely surgery, radiation therapy and chemotherapy, with some exceptions are limited in their ability to treat metastatic and minimal residual disease to cure the patient. Furthermore, the benefit of these conventional therapies is often limited by collateral damage to normal tissues. Immunotherapy is a new avenue of cancer treatment being investigated by researchers and clinicians for a range of different cancer types. It is now understood that the immune system is capable of recognizing and eliminating cancer cells, but that tumors evade and suppress host immune responses and thus persist and spread [1-3]. Immunotherapy seeks to use the patient's own immune system to eliminate cancer cells by overcoming tumor-mediated immune dysfunction and activating cell-mediated immune responses [4]. Such an approach holds great promise for reducing damage to collateral tissue by taking advantage of the inherent specificity of the human immune system. Systemic trafficking and monitoring by immune cells also provide for superior treatment of metastatic and inoperable lesions compared with external beam radiation and surgical therapies. Perhaps most importantly, the generation of immunologic memory following a robust antitumor immune response prevents the recurrence of tumors and can lead to a complete and lasting cure [1].

Approaches to immunotherapy are diverse and can be classified as immune stimulatory or tolerance reversing. Immune-stimulatory approaches aim to improve effector cell maturation and activation, and can increase antigen priming and trafficking of immune cells to lymphoid tissues and the tumor microenvironment. These include cancer vaccines to boost tumor-specific antigen priming $[5,6]$, and the use of engineered cytotoxic T cells (CTLs) [7] and immunocytokines to boost effector-cell survival and activation $[8,9]$. Tolerance-reversing approaches aim to decrease tumor-associated immune tolerance mechanisms such as immune suppressor cells, immune inhibitors and enzymes such as indoleamine 2,3-di-oxygenase [10] and death ligands [2]. In addition, methods to alter the tumor microenvironment using fusion proteins that deliver immunomodulatory molecules (chemokines, costimulatory ligands and adjuvants), can also provide an effective means of reversing tolerance in entrenched tumors [8,9,11-14].
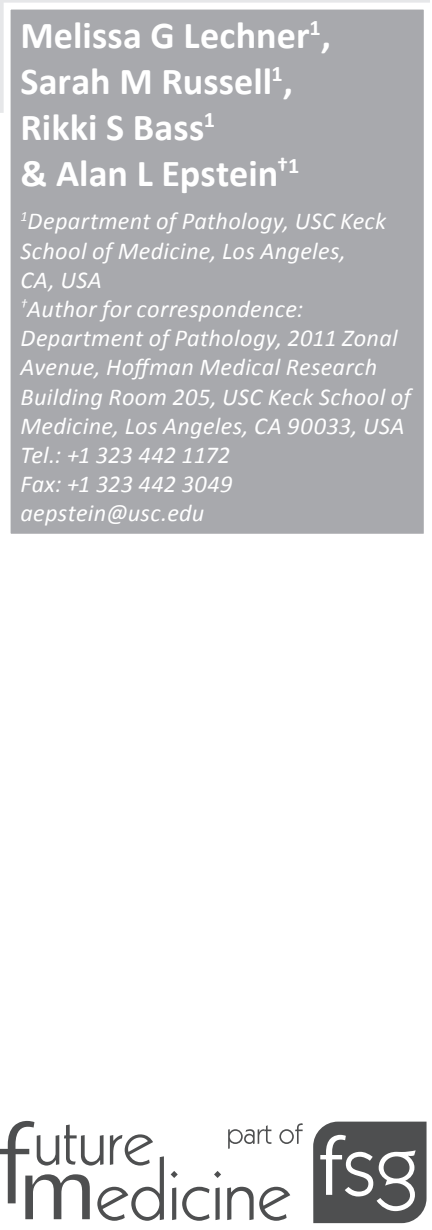
Successful and safe cancer treatments will probably involve a combination of these approaches so that immunostimulatory vaccines or agonist antibodies and ligands can exert their effect in an environment free of immunosuppression $[1,3,15]$.

\section{Chemokines}

One approach to improving antitumor immunity is to increase the infiltration of immune cells into the tumor and facilitate the movement of antigen-presenting cells (APCs) to tumor-draining lymph nodes to prime naive $T$ and $\mathrm{B}$ cells. Chemokines are the endogenous regulators of immune-cell trafficking and homeostasis. They comprise a group of small proteins $(7-15 \mathrm{kDa})$ sharing significant structural homology (C, CC, CXC and CX3C) and acting in gradients to control cell trafficking throughout the body [16-19]. In addition, chemokines may be classified by their expression pattern: homeostatic, expressed constitutively, or inducible, where for example they are upregulated by inflammation [18-21]. A further subset of CXC chemokines with a conserved ELR domain (with the exception of CXCL12 that is ELR-) promotes angiogenesis by attracting endothelial progenitor cells from the bone marrow; ELR CXC chemokines have an angiostatic function [22]. Chemokines are both pleotropic and redundant in their effects and expression and, mediate signals through a group of 20 chemokine receptors. As regulators of cell migration, chemokine networks are frequently usurped by cancer cells to facilitate tumor growth and metastasis by controlling leukocyte tumor infiltration, suppressing antitumor immune responses, regulating angiogenesis and influencing the formation and spread of metastases $[19,20]$. Researchers and clinicians also have been able to use chemokines in immunotherapy to boost immune-cell trafficking to the tumor microenvironment and lymphoid tissues. Some of the most promising chemokine networks for cancer immunotherapy are CCL21-CCL19CCR7; CCL2, CCL3, CCL5 and their cognate receptors; CCL16 (liver-expressed chemokine [LEC]); and CXCL12-CXCR4-CXCR7.

\section{CCR7 \& its ligands CCL21 \& CCL19}

The chemokine receptor CCR7 and its ligand CCL21 (also known as SLC, 6Ckine, exodus-2, Ck $\beta 9$ and TCA-4) and CCL19 (also known as ELC, exodus-3 and Ck $\beta 11$ ) were first identified for their homeostatic role in directing the migration of mature dendritic cells (DCs) from the periphery to tumor-draining lymph nodes for antigen presentation to naive $\mathrm{T}$ cells, which also use CCR7-mediated mechanisms to enter the T-cell zone [23]. These chemokines also have been shown to chemoattract $\mathrm{B}$ cells and NK cells to the lymph nodes. More recently, ectopic CCL19 and CCL21 expression in the tumor microenvironment has been used to bring together naive lymphocytes and mature DCs in a pseudo lymph node for cancer immunotherapy [11]. In 2000, the first studies using recombinant CCL21 as a monotherapy for preclinical tumor models demonstrated a potent immune-mediated antitumor response that led to complete eradication of lung carcinoma tumors [24]. This response was found to be $\mathrm{CD}^{+}$and $\mathrm{CD} 8{ }^{+}$lymphocyte dependent with significant $\mathrm{DC}$ infiltration into tumors and tumor-draining lymph nodes observed. Similar studies by Vicari et al. found that mouse CCL21 exerted antitumor effects through its angiostatic effect and by its activation of $\mathrm{CD} 8^{+} \mathrm{T}$ and possibly NK cell-mediated mechanisms leading to reduced implantation of CCL21 transduced CT26 colon carcinoma cells [25]. Furthermore, CCL19-transduction of murine breast carcinoma cells led to the rejection of tumors in a $\mathrm{NK}$ and $\mathrm{CD}^{+}{ }^{+}$T-cell-mediated manner [26]. In addition to its use as a monotherapy, CCL21 has been included in combined immunotherapy protocols. Studies using murine B16 melanoma lysate-pulsed DCs modified to produce CCL21 demonstrated the ability of this chemokine to enhance the antitumor effects of DC vaccination $[27,28]$. Tumor growth inhibition was significantly better with CCL21-expressing DCs as compared with control DCs or CCL21 alone [27,28]. Furthermore, CCL21-expressing DCs injected into growing tumors were able to recruit and prime naive $T$ cells by creating a lymph node-like structure within the tumor microenvironment [27,28]. Curiously, a recent study by Shields et al. found that CCL21 expression by murine B16-F10 melanoma tumors contributed to tumor immune tolerance, while CCL21 tumors were found to induce antigenspecific immunity [29]. Dubinett et al. have suggested that this discordant result may be attributed to multiple modifications introduced into the tumor model in addition to overexpression of CCL21 [30].

These promising preclinical results have led to ongoing Phase I clinical trials in melanoma at the Moffitt Cancer Center (FL, USA) [30] and in non-small-cell lung cancer (NSCLC) at the University of California Los Angeles (CA, USA) [31] using CCL21-transduced 
DCs (both in collaboration with the National Cancer Institute - Rapid Access to Intervention Development program). In these studies, chemotherapy-naive metastatic melanoma patients are receiving escalating doses of adenoviral CCL21-transduced DCs matured ex vivo and pulsed with MART-1/gp100/NY-ESO-1 peptides. Preliminary results from 12 patients demonstrate accumulation of $\mathrm{CD}^{+}{ }^{+} \mathrm{T}$ cells, but not NK or B cells in biopsies taken of one of several injection sites [30]. Stage IIIb/IV NSCLC patients are receiving intratumoral administration of autologous CCL21-transduced DCs [31]. Owing to the difficulty associated with preparing autologous CCL21-transduced DCs for clinical use, Kar et al. have also developed a novel CCL21-vault nanocapsule for intratumoral delivery of CCL21 to be used in future clinical trials [32].

CCR7-expressing tumor cells are associated with a poor prognosis and nodal metastases. In addition, tumor cells expressing CCR7 generate transcellular gradients of CCL19 and CCL21, therefore promoting tumor-cell migration towards CCL21-expressing lymphatics [33,34]. This relationship was supported by Wiley et al. in a study that demonstrated B16-CCR7injected mice had a 700-fold increase in tumorcell metastasis to draining lymph nodes as compared with controls, CXCR5-B16 cells, 1 week after injection [35]. CCR7 also plays a role in tumorigenesis. Fang et al. demonstrated that CCR7 helps promotes tumorigenesis by downregulating IFN- $\gamma$ in mice inoculated with B16-CCR7 cells compared with mice inoculated with B16 cells alone [36]. Similarly, Muller et al. demonstrated that signaling through CCR7 in breast cancer cells promotes actin polymerization, pseudopodia formation, chemotaxis and invasion [37]. Consistent with these findings, more recent studies have shown CCL19 and CCL21 to be significantly higher in lymph node-positive breast cancer patients than lymph node-negative patients [38]. The role of the CCR7-CCL19/CCL21 axis in lymph node metastasis has been demonstrated in a variety of solid tumors, including melanoma, colorectal cancer, gastric carcinoma, esophageal squamous-cell carcinoma, NSCLC, oral and oropharyngeal squamous-cell carcinoma, squamous-cell carcinoma of tonsil, squamouscell carcinoma of the head and neck, thyroid carcinoma, hepatocellular cancer, prostate cancer and cervical cancer, as well as various hematopoietic malignancies [33,39]. Despite the clear role for CCR7 signaling in tumor metastasis, to date very few studies have investigated the therapeutic effects of blocking CCR7-mediated tumor metastasis. Murine studies of B16 melanoma demonstrate gross metastasis to draining lymph nodes of CCR7-expressing tumors as compared with CCR7 ${ }^{-}$controls. This effect was abrogated by the use of neutralizing anti-CCL21 antibodies [35] demonstrating the potential for targeting this metastasis promoting signaling pathway. The competing roles of CRR7 and its ligands in malignancy demonstrate the need for a careful understanding of this complex interaction before successful chemokine-based immunotherapy can be achieved.

In addition to chemoattraction, CCL21 has been shown to have a variety of other antitumor effects, which include inducing apopotosis resistance in DCs, enhancing tumor sensitivity to subsequent chemotherapy and promoting the survival and cytotoxic activity of transfected T cells [30]. There is also considerable cross-talk between the various chemokine ligand-receptor pairs. Studies using CCL21 gene-modified DCs demonstrated that CCL21 promotes the CXCR3-CXCL9-CXCL10 axis and that neutralization of the CXCR3 ligands inhibited the antitumor responses in these models [30].

\section{CCL16}

The human chemokine CCL16, also known as LEC, promotes the migration and activation of myeloid cell populations to augment the cytotoxic activities of effector T cells [37]. CCL16 binds chemokine receptors CCR1, CCR2, CCR5 and CCR8 to act as a chemotactic factor for monocytes/macrophages, DCs and lymphocytes in the periphery [40,41]. CCL16 is upregulated by inflammatory mediators such as IL-10, suggesting that its physiological role may be to recruit immune cells to peripheral sites of inflammation or infection. CCL16-activated macrophages have increased phagocytic activity and overexpress costimulatory markers (CD80, CD86 and CD40) [37]. Through its indirect effects on APCs and direct effects on effector cells, CCL16 augments the cytotoxic activity of $\mathrm{T}$ effector cells concurrent with increased TNF- $\alpha$ and Fas ligand expression [40]. These physiologic functions of CCL16 suggested its use in cancer immunotherapy to improve immune cell recruitment to the tumor site and increase APC maturation and T-cell priming [40].

The potential therapeutic applications of CCL16 were first studied by Giovarelli et al. [42], who demonstrated mammary carcinoma TSA cells engineered to express CCL16 
inhibited the metastatic spread of tumor and induced tumor rejection owing to an impressive infiltration of macrophages, DCs, T cells and neutrophils, as well as increased production of IFN- $\gamma$ and IL-12. The immunotherapeutic potential of a tumor-targeted LEC fusion protein (LEC-chTNT-3) previously generated in our laboratory has also been demonstrated in murine solid tumor models [43]. As shown in FIGURE 1, treatment with LEC-chTNT-3 chemoattracts lymphocytes, neutrophils, DCs and B cells into the tumor and generates a $40-60 \%$ reduction in well-established solid tumors including MAD109, COLON26 and RENCA [43]. The ability of CCL16 to markedly improve the recognition of poorly immunogenic tumor cells by promoting APC-T-cell interactions establishes it as a prime candidate for targeted immunotherapy. In addition to its chemoattractive properties, LEC fusion proteins also were found to induce vascular thrombosis to cause massive cell death and necrosis in tumors [43]. This property of some chemokines has been described and may be owing to its binding to receptors present on the vasculature in immune activated sties. Additional studies to demonstrate the effect of LEC on tumor vasculature are ongoing, since it is likely that LEC fusion proteins induce the expression of adhesion molecules on tumor endothelial cells to enable immune-cell infiltration required to reverse tolerance and implement tumor destruction within the tumor microenvironment.

\section{CCL2, CCL3 \& CCL5}

The chemokines CCL2 (monocyte chemotactic protein-1), CCL3 (macrophage inflammatory protein-1 $\alpha$ ) and CCL5 (RANTES) have overlapping roles in regulating the migration of multiple subsets of innate and adaptive immune cells. Upon binding of CCL2, CCL3 or CCL5, to their cognate receptors (CCR2, CCR1 and CCR5, respectively), immature DCs, monocytes and memory and $\mathrm{T}$ effector cells extravasate from the vasculature and enter peripheral sites of inflammation or infection [44-46]. The broad chemotactic actions of these proteins have made

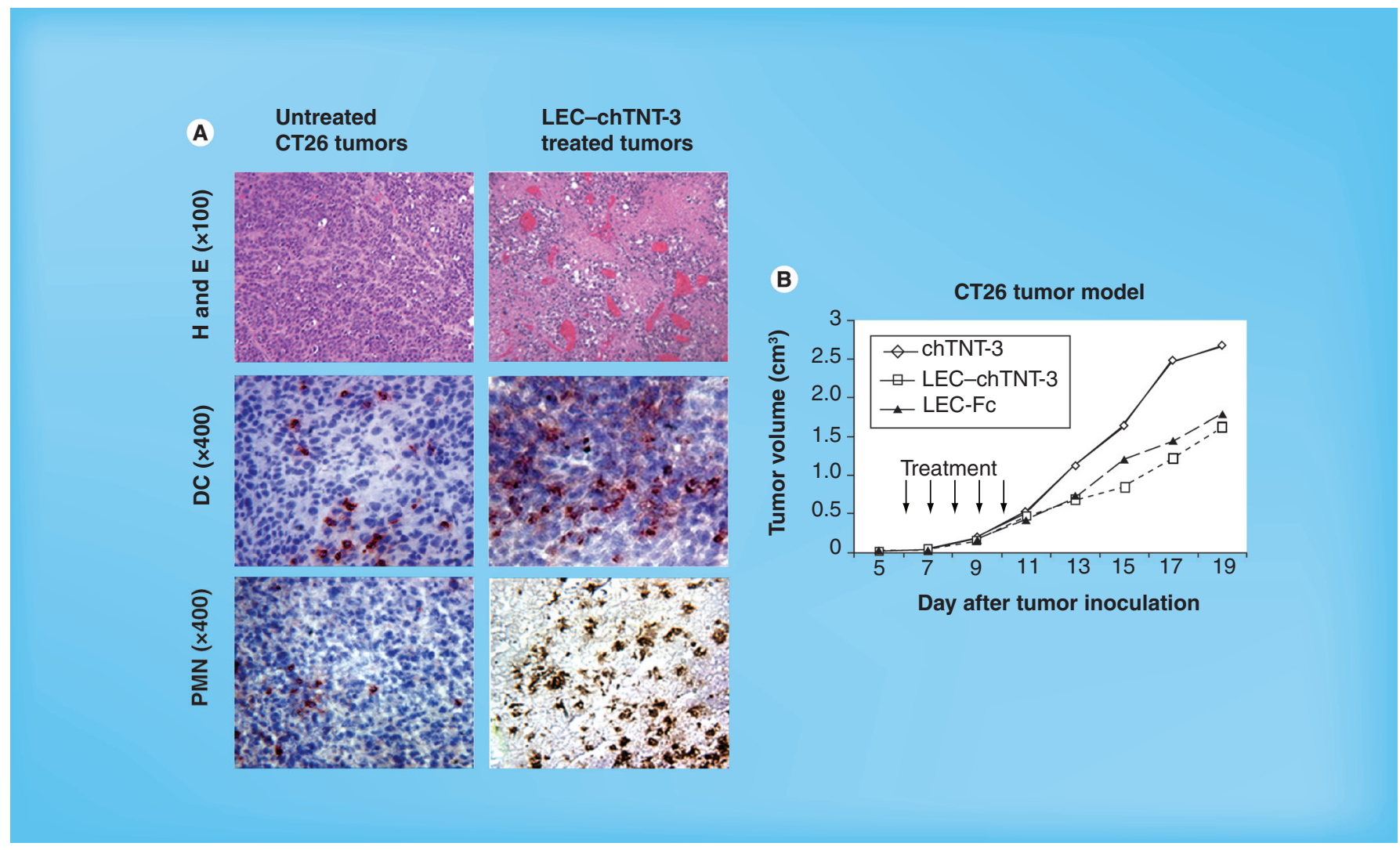

Figure 1. CCL16 immunotherapy increases tumor infiltration by immune cells and inhibits growth of established tumors.

(A) CCL16 treatment of CT26 tumors produced significant influx of immune cells into the tumor site, most notably DCs and granulocytes (original magnification $\times 200$ ). (B) Both targeted (LEC-chTNT-3) and untargeted (LEC-FC) CCL16 fusion proteins significantly inhibited CT26 tumor growth in BALB/C mice. For these studies, tumor-bearing mice $(n=5)$ were injected intravenously with $25 \mu \mathrm{g}$ of LEC reagents daily for 5 days starting on day 6 after tumor implantation.

DC: Dendritic cell; E: Eosin; H: Hematoxylin; LEC: Liver-expressed chemokine; PMN: Polymorphonuclear cell; TNT: Tumor necrosis therapy. 
them important components of cancer immunotherapy strategies aimed at increasing immunecell infiltration into tumors. To this end, CCL2, CCL3 and CCL5 used in monotherapy or in combination therapy, have been shown to induce both tumor regression and immunity to subsequent tumor challenge in multiple preclinical models, as described later [11]. While these chemokines improve tumor infiltration by immune effector cells, they also contribute to the recruitment of suppressor cells that can hamper antitumor immune responses and promote tumor tolerance. CCL2 and CCL3 have been shown to increase the infiltration of Tregs, myeloidderived suppressor cells (MDSCs) and tumorassociated macrophages [47-50]. In addition, CCL2 was shown to be overexpressed by tumorassociated fibroblasts in breast cancer and greater CCL2 and CCL5 levels in the tumor microenvironment correlated with accumulation of tumor associated monocytes and more advanced disease [46]. Similarly, Zhang et al. demonstrated multiple roles for CCL2 in promoting prostate cancer growth, including modulation of tumorassociated macrophage migration and promotion of osteoclast maturation, as well as direct effects on prostate cancer cell proliferation, migration and invasion [51].

Despite the conflicting roles for these chemokines in the tumor microenvironment, numerous preclinical studies have demonstrated their efficacy in tumor immunotherapy. Parker $e t$ al. showed enhanced tumor growth inhibition and greater levels of $\mathrm{CD}^{+}{ }^{+}$and $\mathrm{CD} 8^{+} \mathrm{T}$-cell infiltrates in murine flank neuroblastoma treated with sequential treatments of HSV-1 expressing IL-12 and HSV-1 expressing CCL2 when compared with either treatment alone [52]. Furthermore, Nagai et al. demonstrated constitutively secreting CCL2 human, malignant glioma vaccinations in nude mice induced tumor infiltration by NK cells and monocytes [53]. Similar results were found in studies using CCL3. Hirose et al. showed that nude mice given subcutaneous injections of Chinese hamster ovary cells genetically modified to secrete CCL3 demonstrated greater tumor growth inhibition and greater neutrophilic infiltration when compared with controls [54]. Cao et al. demonstrated that CCL3 recruited DCs, transduced with a tumor antigen gene, induced a strong CTL response and effectively eliminated established tumors and prevented metastases [55]. CCL5 was also found to be effective when used as a monotherapy or in combination immunotherapy protocols. Aravindaram et al. demonstrated
B16/gp100 primary tumors and lung metastasis in C57BL/6JNarl mice are strongly suppressed in murine models treated with gp100 vaccination and CCL5 therapy, which induces more potent splenocyte cytotoxic activities toward B16/gp100 cells [56]. Higher levels of IL-4, IL-6, IFN- $\gamma$ and TNF- $\alpha$ along with longer survival times are seen in mice treated with recombinant CCL5 protein and GM-CSF-transduced tumor cell vaccines when compared with mice treated solely with GM-CSF-transduced vaccines [57]. CCL5 and FLT3L combined with a DNA vaccine has also been shown to inhibit tumor growth in hepatitis B viral antigen $\mathrm{HBc}$ expressed B16 melanoma model [58]. Lapteva et al. created an Ad-RANTES-E1A vaccine, which utilizes a recombinant oncolytic adenovirus expressing CCL5 that induces primary tumor regression and blocks metastasis in JC mammary carcinoma murine models [44].

While CCL2, CCL3 and CCL5 certainly play a role in cancer progression and malignancy, these chemokines may also act as adjuvants in cancer immunotherapy for solid tumors owing to their ability to increase tumor-site immune-cell infiltration. In light of the contrasting roles of CCL2, CCL3 and CCL5 in the tumor microenvironment, immunotherapeutic strategies using depletion or inactivation of suppressor cell populations in addition to chemokine-based stimulation of antitumor immunity may prove especially effective.

\section{CXCL12-CXCR4-CXCR7}

It would be remiss to not touch upon the CXCL12-CXCR4-CXCR7 chemokine axis here given its recently recognized importance in cancer metastasis, but to date successful therapies inhibiting these interactions have been limited. In addition to promoting angiogenesis as discussed above, CXCL12 regulates trafficking of immature and maturing immune cells, namely monocytes, $T$ cells and neutrophils, to and from the bone marrow to body tissues for immune surveillance [59,60]. CXCR4 was first identified as the receptor for chemokine CXCL12; however, a second receptor, CXCR7, has also been identified [61]. These two receptors have been shown to have both overlapping and distinct functions and signaling pathways [61]. While under noninflammatory or low-inflammatory conditions, CXCL12 attracts monocytic cells and $T$ cells to various tissues as a part of immune policing, expression of CXCL12 in the setting of tumor, inflammation has immunosuppressive effects [59-62]. In the tumor microenvironment, CXCL12 functions as 
an anti-inflammatory chemokine that skews the polarization of antigen-specific Tregs and IL-10producing DCs/monocytic cells to restrain the inflammatory process and suppress antitumor immunity in cancerous diseases [61,63].

Furthermore, cancers use the homing function of CXCL12 and its receptors to their advantage, to targeting organs for metastasis, particularly to bone and brain [60]. Cancers including breast, prostate, lung (small-cell and non-small-cell), pancreatic, head and neck, bladder, renal cell, ovarian and melanoma [61] have overexpression of CXCL12 receptors CXCR4 and/or CXCR7 while the corresponding normal tissues show low or absent expression. In prostate cancer, Sun et al. demonstrated blocking CXCL12-CXCR4 interaction with a neutralizing antibody or blocking peptide reduced bone metastases in a murine model [64]. Additional evidence for the role of CXCL12-CXCR4-CXCR7 in metastasis comes from breast cancer models where Liang $e t$ al. inhibited bone metastasis by siRNA-mediated knockdown of CXCR4 [65] and work by Miao et al. [66] and Salmaggi et al. [67] established that expression of CXCR7 on breast cancer cells facilitates spread to lung and brain tissues, respectively. Furthermore, Lee et al. demonstrated inhibition of CXCR 4 blocks metastatic implantation of CXCR4-transduced B16 melanoma cells in lung and that in established lung metastases low doses of cyclophosphamide combined with CXCR4 inhibition yields a $70 \%$ reduction of B16 metastases in the lungs when compared with cyclophosphamide alone [68]. In addition, this work demonstrated that CXCR4 blockers work synergistically with cyclophosphamide or an anti-CTLA-4 monoclonal antibody [68]. Of interest, upregulation of CXCR4 or CXCR7 on cancer cells may be driven by hypoxia and HIF1 $\alpha$ signaling, a common feature of the tumor microenvironment that is known to contribute to other immune defects [69-73].

While significant advances have been made in elucidating the role of CXCL12-CXCR4-CXCR7 signaling in tumor progression, therapeutic modulation of this chemokine axis remains a challenge. Because of the role of CXCR4 in HIV tropism and viral entry, it has been the focus of drug development and virology has yielded a number of CXCR4 antagonists, including a 14-mer peptide (TN14003) [65], bicyclams (AMD3100) and T22, TN14003, CTCE-9908 and ALX40-4C, which are analogs and peptides designed to the amino-terminal region of the chemokine, CXCL12 [61]. In addition to their role in HIV, some of these antagonists have been found to have other therapeutic uses. Specifically, AMD3100 (Plerixafor) has been approved for use in combination with G-CSF for the mobilization of CD34 ${ }^{+}$hematopoietic stem cells for autologous transplantation after high-dose chemotherapy for multiple myeloma and non-Hodgkin's lymphoma [74]. CXCR7 inhibitors have also been developed; some small-molecular inhibitors such as CCX733 or CCX266, siRNA and blocking antibodies are already employed in experimental models in vitro and in vivo [75]. Sun et al. reported that single inhibition of CXCR4-CXCL12 without addressing CXCR7 produced only partial responses and that simultaneous inhibition was probably required to achieve clinically relevant results [61]. Furthermore, the role of CXCL12 in tumor progression is complex and optimal manipulation of CXCL12 for cancer immunotherapy will require a comprehensive understanding of its diverse functions. While CXCL12-CXCR4-CXCR7 interactions contribute to immunosuppression and metastasis, they also facilitate tumor immune surveillance by regulating trafficking of immature and maturing immune cells and promoting angiogenesis.

Chemokines play a major role in antitumor immune responses and in tumor immune escape. As such they hold great potential in cancer immunotherapy for increasing immunecell infiltration of the tumor microenvironment to facilitate productive immune interactions (FIGURe 2A). Their beneficial effect of improving immune-cell infiltration is countered by their contribution to recruitment of immune suppressor cell populations and tumor metastasis (Figures 2A \& B). Studies in experimental tumor models and cancer patients clearly demonstrate the potential of chemokine immunotherapy and suggest that future trials should seek to incorporate chemokines into therapy protocols.

\section{T-cell costimulatory molecules}

In addition to facilitating immune cell interactions and infiltration through chemokine modulation, immunotherapy can also augment antitumor immunity by increasing T-cell priming by APCs. Tumor and endogenous antigens are carried to lymphoid tissues by APCs via the lymphatic system where they are presented to naive effector cells. For optimal T-cell priming, T-cell receptor (TCR) recognition of MHCpresented antigen (signal one) must be accompanied by costimulatory signals, namely ligation of T-cell CD28 with APC B7.1 (CD80) or B7.2 (CD86) (signal two) [76]. The absence of the second costimulatory interaction leads to T-cell 


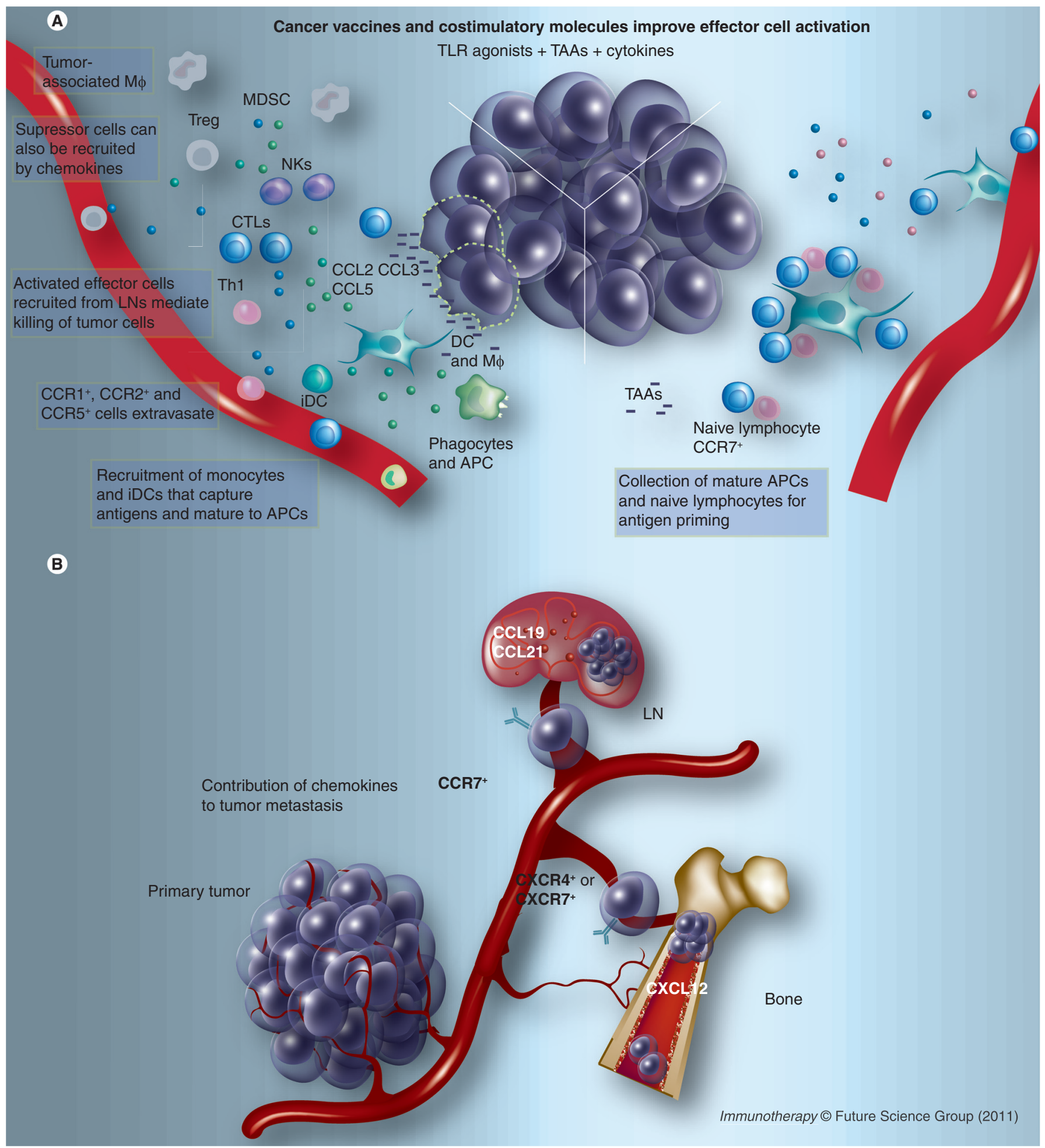

Figure 2. Chemokines for cancer immunotherapy and their role in cancer metastasis. (A) Chemokine immunotherapy to promote naive and activated immune-cell infiltration into the tumor microenvironment. One challenge of using chemokines for immunotherapy is their dual actions in recruiting immune effector and immune suppressor cells. Chemokine immunotherapy may be paired with strategies to boost tumor antigen capture and presentation for robust antitumor immunity. (B) Aberrant overexpression of chemokine receptors mediates tumor cell migration and seeding of metastatic disease. CCL19 and CCL21 facilitate homing of naive lymphocytes and mature APCs to LNs under normal conditions, but can be used by CCR7+ tumor cells to migrate to tumor draining LNs. CXCL12 normally regulates immune cell homeostasis in and out of the bone marrow and is co-opted by CXCR4 ${ }^{+} / C X C R 7^{+}$tumor cells for metastasis to bone.

APC: Antigen presenting cell; DC: Dendritic cell; iDC: Immature dendritic cell; LN: Lymph node; M $\phi$ : Macrophage; MDSC: Myeloid-derived suppressor cell; TAA: Tumor-associated antigen; Th: T helper cell; TLR: Toll-like receptor. 
undocking from the APC (APC-T-cell priming failure) and subsequent anergy. A common immune defect observed in cancer patients and experimental tumor models is immature APCs that fail to express T-cell costimulatory ligands and subsequently elicit poor T-cell activation [4]. Furthermore, studies show that the weak immunogenicity of tumor antigens might also be owing to inappropriate or absent expression of costimulatory molecules on tumor cells [77]. One immunotherapy approach in our laboratory and others is to provide artificial costimulatory signals in the form of agonist antibodies or, preferentially, immunoligand fusion proteins [12-14,78].

\section{B7 ligands}

B7.1 and B7.2 are prototypic costimulatory molecules and have the ability to provide $\mathrm{T}$ cells with the second signal that ensures T-cell activation supersedes T-cell anergy. Several strategies have successfully used B7 for the immunotherapy of solid tumors and hematologic malignancies, including tumor cell expression of B7 and soluble B7-immunoglobulin fusion proteins. In a key study by Townsend and Allison, B7-transfected K1735 melanoma cells were rejected (9/10) effectively in mice compared with aggressively growing wild-type tumors [79]. Tumor rejection of B7-melanoma cells in these mice occurred by a $\mathrm{CD} 8^{+} \mathrm{T}$-cell dependent mechanism and provided significant protection to rechallenge with the wild-type tumor. These results were confirmed by others using B7 transfection of murine melanoma [80], colorectal [80] and mammary [81] tumor models. In more recent work, viral-mediated tumor expression of B7 in combination with other costimulatory ligands ICAM-3 and LFA-3 has shown promise in preclinical and clinical trials for the treatment of chronic lymphocytic leukemia [82] and metastatic melanoma [83]. In the late 1990s, the generation of soluble B7 molecules was studied and found to be very effective for the immunotherapy of solid tumors [84,85]. Sturmhoefel et al. demonstrated complete regression of 7-day old tumors in three murine models (MethA, P815 and MB49) and partial response in poorly immunogenic B16 melanoma tumors by treatment with soluble B7.1-Fc or B7.2-Fc fusion proteins [85]. More recently, our laboratory has generated two B7 fusion proteins (B7.1-Fc and B7.2-Fc) that significantly inhibited growth of COLON26, RENCA and WeHi tumor models in mice as single agent immunotherapy in a dose-responsive manner (FIGURE 3) $[78,86]$. Other strategies to express these costimulatory molecules on tumor cells include those by McHugh et al. who constructed a recombinant glycol-lipid-anchored protein attached to the extracellular domain of human B7.1 [87]. This fusion protein was inserted in the tumor cell membrane and provided the necessary signal for the proliferation of CTLs in a mixed lymphocyte reaction. As expected, B7 immunotherapy studies have consistently shown greater treatment efficacy (as defined by parameters such as complete tumor regression, systemic immune reaction and immunologic memory) against strongly-immunogenic tumor models than poorly immunogenic ones [85,88] and when combined with other methods of immune stimulation: the cytokines IL-12 [80,89], GM-CSF [80] or IFN- $\gamma$ [81]; tumor vaccines [85]; and costimulatory ligands [82,83,90]. In addition, combined B7 immunotherapy with radiation therapy [89] or chemotherapy [91] - two methods that increase tumor necrosis and endogenous antigen presentation - produced greater antitumor responses than monotherapy, a theme that appears across costimulatory molecule therapies. These data suggest that the use of B7 costimulatory molecules might be a promising novel approach for cancer immunotherapy. Since, however, B7.1 binds the negative inhibitor CTLA- 4 more strongly than its agonist TCR CD28, its use may be limited to those tumors that do not express or induce CTLA- 4 .

\section{T-cell costimulatory molecules \& receptors}

Beyond canonical signal one, interactions between T-cell costimulatory molecules expressed on activated APCs and their cognate receptors on activated $T$ cells strengthen and sustain effector responses [12-14,92]. Figure 4 illustrates the complex interaction between $\mathrm{T}$ cells and APCs in the immunologic synapse that results in robust immune stimulation. These interactions enhance the proliferation, cytokine response and survival of activated effector $T$ cells and are critical for the generation of immunologic memory [12-14,78]. In contrast to B7 ligands, which act on naive lymphocytes, these T-cell costimulatory ligands act to boost responses of antigen-primed $\mathrm{T}$ cells and their receptors specifically and are typically upregulated following activation with TCR and CD28 signaling [12-14].

T-cell costimulatory molecules in the TNF super family (TNFSF) play a particularly important role in cell-mediated immune responses and as such have been the focus of much research in cancer immunotherapy. TNFSF receptors 

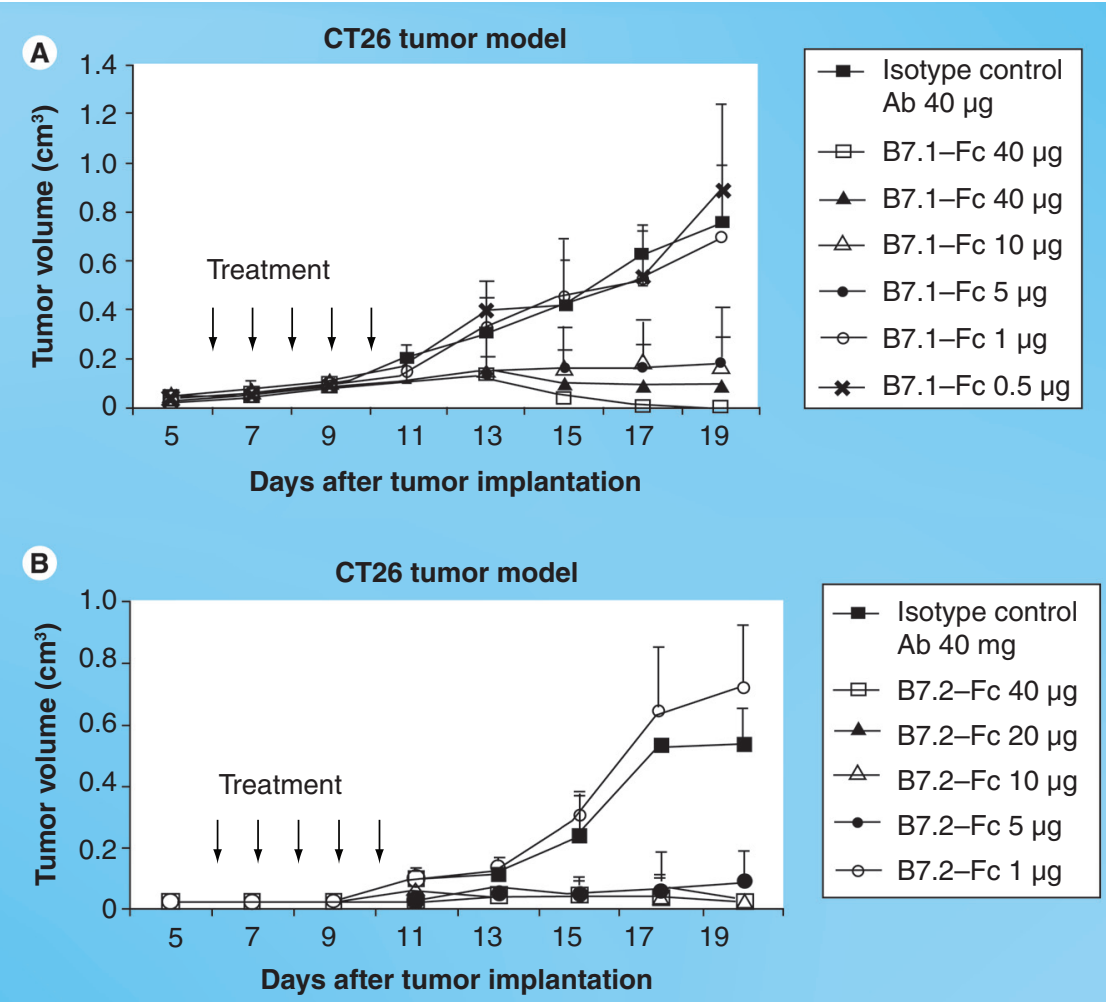

Figure 3. Dosing studies of B7.1-FC and B7.2-FC immunotherapy in the CT26 tumor model. In these in vivo studies, both (A) B7.1-FC and (B) B7.2-FC showed a dose response effect to inhibit tumor growth in this murine solid tumor model. All mice $(n=5)$ received five daily intravenous treatments starting on day 6 after tumor implantation. Ab: Antibody.

and their ligands play an important role in the proliferation, differentiation, activation and survival of both tumor and immune effector cells. Broadly, members of this group induce apoptosis (Fas-L, TRAIL-R and TNF-R) or regulate $\mathrm{B}$ and $\mathrm{T}$-cell immune responses (OX40-L, CD40-L, glucocorticoid-induced TNFRrelated protein [GITR]-L and 41BB-L). The latter group is of interest for T-cell costimulatory immunotherapy and shares significant structural homology, including type 1 transmembrane protein receptors with a cysteine-rich extracellular domain and type II transmembrane protein ligands that often are biologically active as homotrimers [12-14]. These latter molecules are critical for APC-T-cell interactions and enhance the structural integrity of the APC-T-cell synapse that leads to T-cell activation and the induction of third signal molecules (IL-2 and IL-12) that expand and license CTLs against tumor antigens. Currently, agonist antibodies for GITR, CD137 and OX40 are in the clinic to provide proof-of-principle that replacement of missing costimulation can significantly enhance effective immunotherapy [12-14]. In the future, fusion proteins of the ligands of these receptors may be tested in parallel especially considering their probable greater safety level, shorter in vivo halflife and ability to simulate normal physiological conditions at the DC-T-cell synapse.

\section{OX40-OX40L}

The OX40 receptor (CD134) is expressed on activated effector $\mathrm{T}$ cells (stably on $\mathrm{CD}^{+}{ }^{+}$and transiently on $\mathrm{CD}^{+}$), but not on naive or resting lymphocytes. It facilitates signaling critical for optimal T-cell proliferation, survival and memory. The OX40 ligand (OX40L) is accordingly expressed on APCs, including DCs, B cells and macrophages, following activation by Tolllike receptor (TLR) agonists (TLR4, TLR2 and TLR9) and CD40-CD40L signaling [14,93]. Signaling through OX40 acts on stimulated $T$ cells to enhance late proliferation and survival and augments cytokine secretion to boost both Th1 and Th2 cell responses (IL-2, IL-4, IL-5 and IFN- $\gamma$ ).

Given the power of OX40-OX40L interactions to boost effector cell responses, numerous groups have tested OX40 agonists and OX40L fusion proteins for the immunotherapy of tumors [14]. The results of these studies clearly show efficacy 


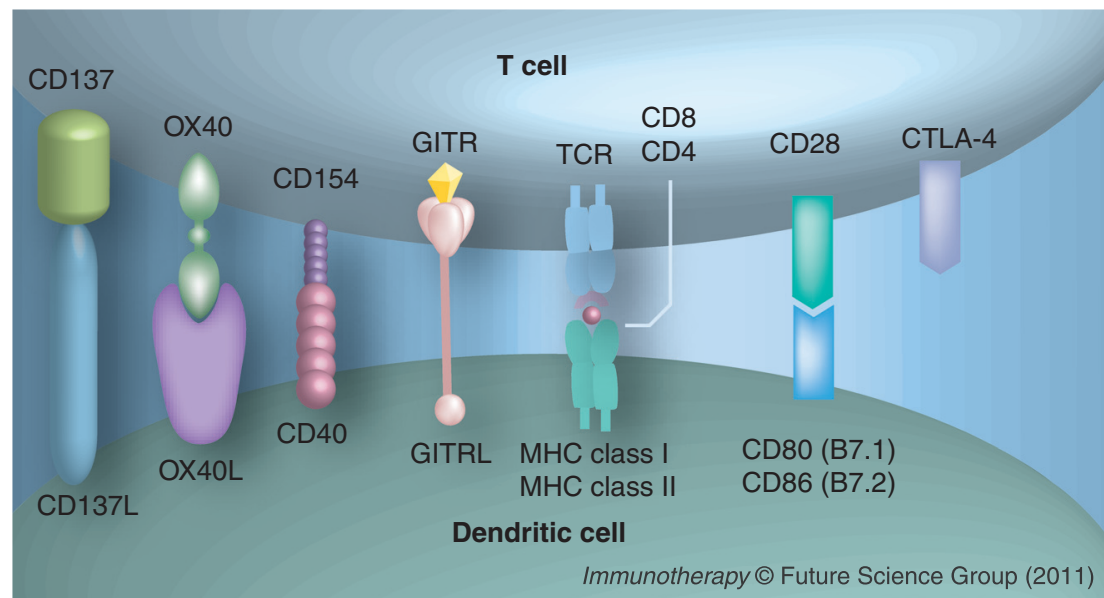

Figure 4. Dendritic cell-T cell synapse. During T-cell docking to dendritic cells, different costimulatory molecules migrate into the synapse to stabilize the cell-cell interaction required for T-cell activation to the peptide presented in the MHC cleft. Without this stabilization, the synapse is too unstable and the cells undock, producing T-cell anergy. CTLA-4 is the inhibitory molecule that, if present, preferentially binds B7.1, thereby preventing T-cell activation.

in inhibiting the growth of established tumors in association with increased frequency and survival of tumor-specific $T$ cells [14,94-98]. In a study by Weinberg et al., treatment of established tumors with either anti-OX40 agonist antibody or Fc-OX40L fusion protein significantly augmented tumor-specific $\mathrm{CD}^{+}{ }^{+} \mathrm{T}$-cell responses and increased survival in a dose-dependent fashion in four murine tumor models (B16 melanoma, MCA sarcoma, CT26 colon carcinoma and SM1 breast cancer) [94]. These findings were supported by subsequent studies showing that treatment with $\mathrm{Fc}-\mathrm{OX} 40 \mathrm{~L}$ inhibited the growth of subcutaneous established tumors and lung metastases of $4 \mathrm{~T} 1$ breast and CT2 6 colon cancers in mice [95] and, when incorporated into tumor cell vaccines, protected mice from subsequent challenge with SM1 breast carcinoma cells [96]. As shown in Figure 5, our laboratory generated a C-terminal OX40L fusion protein ( $\mathrm{Fc}-\mathrm{mOX} 40 \mathrm{~L})$ that produced complete regression of established tumors in CT26 colon and RENCA renal cell cancer models and showed greater efficacy than the anti-OX40 agonist antibody OX86 [97].

The ability of OX40 agonists to protect appears to correlate with the immunogenicity of the tumor [99], in that poorly immunogenic tumors do not respond to OX40 agonist treatment, whereas tumor protection is achieved in immunogenic models where endogenous antigen priming occurs and stimulates induction of OX40 receptors on tumor-specific $T$ cells. This is consistent with action on $T$ cells following robust TCR signaling rather than action on naive $T$ cells. Furthermore, this suggests that OX40 agonist treatment would be beneficial when given after vaccination or other antigen-priming therapy in poorly immunogenic models. Indeed, Murata et al. showed in a murine breast cancer model that combination of a GM-CSF-secreting tumor cell vaccine with OX40 agonist treatment produced greater expansion and prolonged survival of tumor-specific $T$ cells compared with vaccine or OX40 treatment alone, yielding greater overall tumor regression [100]. Immunotherapy of $4 \mathrm{~T} 1$ breast and CT26 colon cancer models with combined Fc-OX40L and GM-CSF tumor vaccine was shown to be more effective at inhibiting tumor growth than either treatment alone by Ali et al. [95]. Similarly, concurrent or preceding treatment with chemotherapy [101] or radiation therapy $[102,103]$ - two strategties that boost endogenous tumor antigen priming - improved efficacy of OX40 agonist therapy for treatment of established tumors in mice. One common observation by laboratories, including our own, is that systemically administered OX 40L fusion proteins or agonist antibodies alter lymphocyte homing and cause transient splenomegaly, a finding now being seen in the clinic.

\section{GITR-GITRL}

Glucocorticoid-induced TNFR-related protein, also known as activation-inducible TNFR family receptor (AITR) is another member of the TNFSF. Similar to OX40, its expression is rapidly upregulated on $\mathrm{CD}^{+}$and $\mathrm{CD} 8^{+}$effector T cells upon TCR/CD28 and effects of signaling relate to the strength of the initial TCR stimulation. The cognate ligand, GITRL, is expressed on both endothelial cells and APCs (DCs and macrophages, transiently on monocytes) and may be aberrantly expressed by a number of solid maligancies and leukemias [12]. A major function of GITR signaling in T effector cells is protection from activation-induced cell death [104]. Furthermore, it increases T-cell proliferation and cytokine production and, in the presence of inflammatory stimuli, simultaneously allows T effector cells to overcome Treg suppression and may augment Treg activity [105]. Interestingly, high levels of GITR are constitutively expressed on Tregs and further upregulated with stimulation, suggesting a role in preventing autoimmune reactions during immune activation [106]. GITR expression has also been reported on macrophages and NK cells following activation though effects of GITR signaling in these populations is less clear [106]. Placke et al. reports that GITR signaling has an inhibitory effect on NK cells, including reduced cytotoxicity and IFN $\gamma$ production, by reducing NF- $\kappa B$ activity. As with OX40, the 
effects of GITR costimulation appear to depend on the strength of initial TCR signaling and the level of concurrent immune activation, suggesting that GITR immunotherapy must be optimized for the level of immune activation present in a given tumor [12].

Numerous groups have shown effective immunotherapy of established murine tumors through enforced GITRL expression, addition of recombinant GITRL, or treatment with agonistic anti-GITR antibodies [107]. GITRL transfection of CT26 tumor cells delayed tumor growth and improved survival in a murine model, with effective therapy accompanied by enhanced tumor infiltration by $\mathrm{CD} 8^{+} \mathrm{T}$ cells and increased effector function [108]. GITRL-expressing cancer cells also show delayed tumor growth in a CD8 ${ }^{+}$T-cell-dependent fashion and effector $\mathrm{T}$ cells in this model overcame Treg-mediated suppression [109]. In a sarcoma model in mice, immunization with a plasmid encoding a tumorspecific antigen and GITRL produced a tenfold increase in tumor-specific $\mathrm{T}$ cells with primary effects mediated by $\mathrm{CD} 8{ }^{+} \mathrm{T}$ cells that become resistant to Treg-mediated suppression. Finally, as shown in Figure 6, immunotherapy studies from our own laboratory using a Fc-mGITRL fusion protein produced regression of CT26 and RENCA murine tumor models concurrent with induction of $\mathrm{CD} 8{ }^{+} \mathrm{T}$-cell proliferation and loss of suppressive function by Treg cells [110]. Taken collectively, these data indicate that GITR immunotherapy is mediated through improved $\mathrm{CD}^{+} \mathrm{T}$-cell infiltration into the tumor microenvironment, proliferation and cytotoxicity (cytokine production, granule mobilization and lytic activity) and inactivation of or resistance to Treg suppression [111].
- CD137-CD137L (41BB-41BBL)

T-cell costimulatory receptor $\mathrm{CD} 137$ (41BB) and its cognate ligand CD137L (41BBL) are induced following immune activation and serve as a key signaling pathway for antigen-primed $\mathrm{CD}_{4}{ }^{+}$and CD8 ${ }^{+} \mathrm{T}$ cells and NK cells. CD137L expressed on mature APCs, including DCs, B cells and monocytes, provides potent survival signals to activated T cells [112-115] and enhances the magnitude (duration) and breadth (epitope spread) of their responses [116]. As observed with GITR-GITRL signaling, CD137-CD137L interactions appear to have more potent costimulatory effects on $\mathrm{CD}^{+}$than $\mathrm{CD}^{+}{ }^{+} \mathrm{T}$ cells [117].

Stimulation of CD137 signaling by CD137 agonist antibodies or CD137L fusion proteins has been shown to improve antitumor immunity and inhibit tumor growth. In an important study by Melero et al., established Ag104A sarcoma and P815 mastocytoma tumors were successfully eradicated by treatment with a CD137 agonist antibody. Tumor regression was mediated by both $\mathrm{CD} 4^{+}$and $\mathrm{CD} 8^{+} \mathrm{T}$ cells and accompanied by a pronounced improvement in tumor-specific CTL activity [118]. In our laboratory, Zhang et al. constructed a soluble Fc$\mathrm{mCD} 137 \mathrm{~L}$ fusion protein $(\mathrm{Fc}-\mathrm{mCD} 137 \mathrm{~L})$ for immunotherapy that similarly inhibited tumor growth in CT26 tumor-bearing mice [119]. These data suggest an important role for stimulation through CD137 on activated effector cells that produces beneficial immune responses in the cancer setting.

Of interest, Miller et al. demonstrated and established the optimal timeline of CD137 agonist therapy as more effective after tumor establishment rather than early on in tumor development [120]. The later period was proposed
A

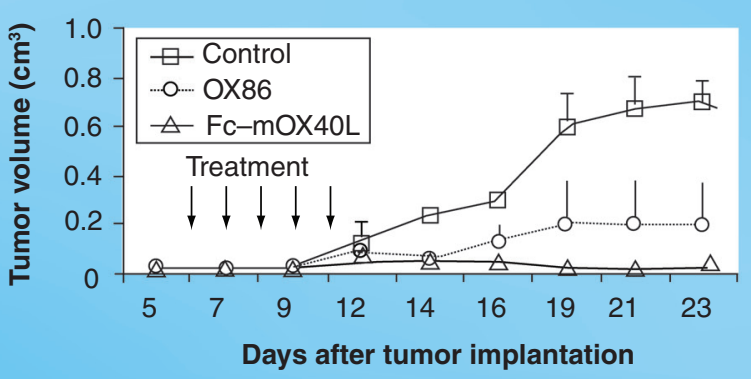

B

RENCA tumor model

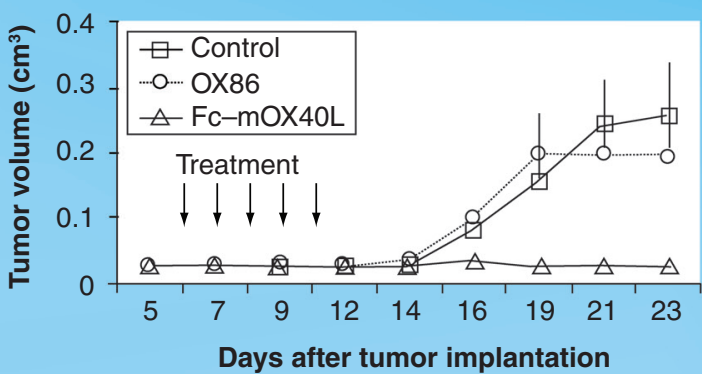

Figure 5. Fc-mOX40L immunotherapy of CT26 and RENCA tumors in mice. These studies demonstrate that both tumor models (A \& B) do not have OX40L costimulation that, when provided, facilitates an effective tumor response by the immune system. OX86, an agonist antibody to OX40, was found to be less effective than the ligand reagents by these methods. All mice $(n=5)$ received five daily intravenous treatments starting on day 6 after tumor implantation. 
A

CT26 tumor model

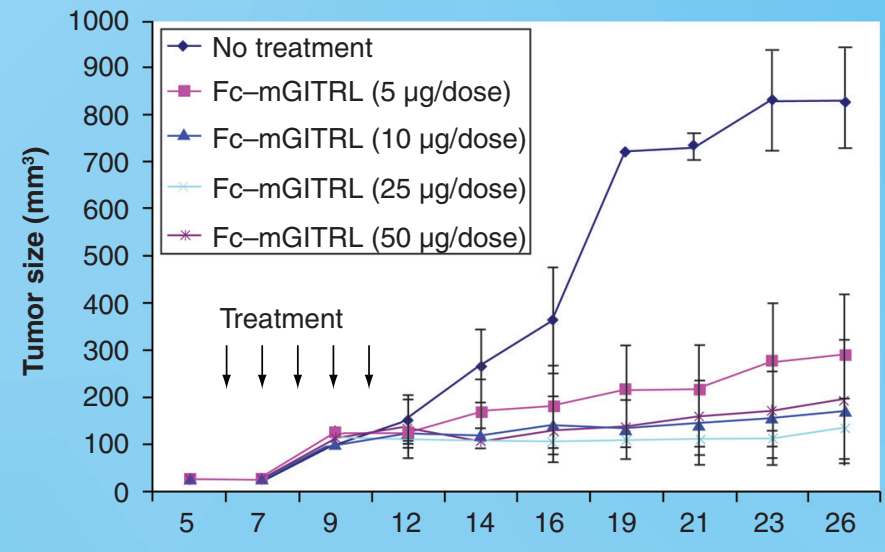

Day post-tumor inoculation

B

RENCA tumor model

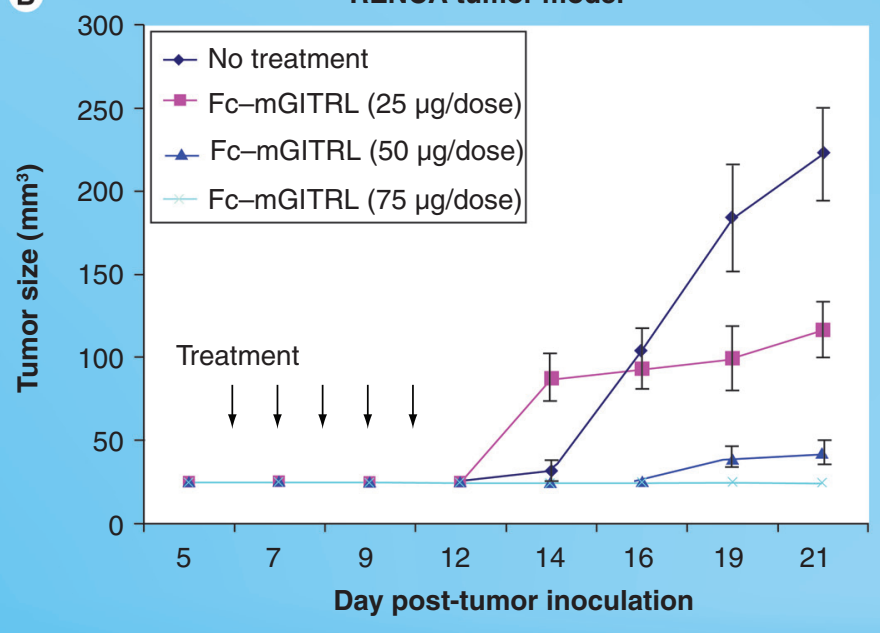

Figure 6. Fc-mGITRL immunotherapy of CT26 and RENCA murine tumor models. Dosing studies of Fc-mGITRL showed a dose response in both tumor models. Based upon these results, the CT26 tumor model (A) was found to be more sensitive to FC-mGITRL treatment than the RENCA tumor model $(\mathbf{B})$ in BALB/C mice. All mice $(n=5)$ received five daily intravenous treatments starting on day 6 after tumor implantation.

to have increased tumor antigen presentation and T-cell activation and suggest that, as seen with OX40 and GITR immunotherapy, effects of CD137 immunotherapy will be augmented when provided in conjunction with antigen priming. Consistent with this notion, treatment of tumor-bearing mice immunized with GM-CSF-transduced tumor cells [121] or a DC vaccine [122] with CD137 agonist antibody treatment produced superior antitumor immunity than either treatment alone. Collectively, the results suggest that enhancing the antigen presentation capacity of the host also enhances the proportion of specifically activated $\mathrm{T}$ cells upon which CD137 agonists can act. Interactions between DCs that have processed antigen and antigen-specific $\mathrm{CD} 8^{+} \mathrm{T}$ cells appear to be both necessary and sufficient to promote priming and activation of the $\mathrm{T}$ cells to a point at which costimulation through CD137 is effective in generating antitumor immunity.

\section{CTLA-4 immunotherapy}

A parallel immunotherapy approach to stimulate T-cell costimulatory ligands is by inhibiting CTLA-4. CTLA-4 is the natural inhibitor of $\mathrm{B} 7$ costimulation and is the critical regulatory molecule for immune privileged sites in the body [123,124]. Two fully human monoclonal antagonist antibodies have been developed for the immunotherapy of solid tumors in patients: ipilimumab and tremelimumab [125]. These 
reagents have been studied extensively in melanoma patients and are now being explored for clinical benefit in other solid tumors, including lung, prostate and renal cell cancer. Despite only marginal improvements in response rates and overall survival compared with conventional chemotherapy regimens, anti-CTLA-4 therapies are encouraging for the long durability of effects in the minority of patients that do obtain partial or complete remission [126]. Early trials comparing tremelimumab to standard dacarbazine or temozolomide chemotherapy for the treatment of melanoma found no significant difference in median overall survival (11.7 vs 10.7 months), complete remissions (1.5 vs $1.8 \%$ ) or partial response rates (7.6 vs 8.3\%) [127]. A Phase II trial with ipilimumab for pretreated melanoma showed a slightly higher response rate (11.1\%) and similar median overall survival at the highest tested dose $(10 \mathrm{mg} / \mathrm{kg}$ four-times every 3 weeks) [128]. Later Phase II studies evaluating ipilimumab $(10 \mathrm{mg} / \mathrm{kg})$ for the treatment of advanced melanoma demonstrated disease control (partial or complete remission or stable disease) in $27-35 \%$ of patients, with an improvement in 2-year survival from 30 to $42 \%$ with ipilimumab treatment compared with $15-20 \%$ for melanoma patients in all cooperative group Phase II studies [128-130]. Most recently, Robert et al. published the results of a Phase III clinical trial combining standard chemotherapy regimen with anti-CTLA-4 monoclonal antibody ipilimumab in previously untreated patients with metastatic melanoma [131]. The results showed a statistically significant improvement in overall survival at 1 ( 47.3 vs $36.3 \%), 2$ (28.5 vs $17.9 \%)$ and $3(20.8$ vs $12.2 \%)$ years, although the clinical relevance of these modest improvements must be evaluated by patients and their providers. Prolonged survival on combination therapy was achieved with a significantly higher rate of grade $3 / 4$ adverse events (56.3 of patients treated with ipilimumab plus dacarbazine, as compared with $27.5 \%$ treated with dacarbazine and placebo). Furthermore, when only immune-related adverse events are examined, the total number of events is more than doubled, and the rate of grade $3 / 4$ adverse events is nearly seven-times greater (41.7 compared with 6\%) with combined anti-CTLA- 4 and dacarbazine therapy compared with dacarbazine alone. Given the importance of immune regulatory mechanisms in terminating physiological immune responses and preventing autoimmune disease, it is not unexpected that a common side effect of antiCTLA-4 therapy is immune-mediated damage of dermal, gastrointestinal, ocular, endocrine and hepatic tissues. Immune-related adverse events (irAEs) with anti-CTLA-4 therapy are considered class-specific (occurring across all indications), dose dependent, directly correlated with the success of immunotherapy and are usually treatment limiting [125,132-134]. Histopathology of gut, ocular and skin lesions shows a clear immune etiology with heavy infiltration of $\mathrm{CD}^{+}$and $\mathrm{CD}^{+} \mathrm{T}$ lymphocytes [125,135]. The frequency of such irAEs with anti-CTLA- 4 therapy is alarming, with the standard dose (10 mg/kg four-times every 3 weeks) of ipilimumab producing drug-related adverse events in $84.6 \%$ of patients (severe or worse in $32.6 \%$ ) with $72.3 \%$ of patients having irAEs (25.2\% grade 3/4) [125]. This rate of adverse effects must be weighed against the improved clinical outcomes (survival and tumor response) and compared with other available treatments, namely chemotherapy regimens.

\section{Targeting immune stimulation to the tumor}

Despite the preclinical promise of chemokines and costimulatory molecules in cancer immunotherapy, responses in patients have been limited in part by traditional administration methods as soluble, systemic reagents. Systemic infusion produces nonspecific activation of immune cells and cytokine release that may lead to significant systemic toxicity and autoimmune reactions. In addition to the anti-CTLA- 4 treatment examples discussed above, systemic IL-2 therapy provides additional evidence of the risks of untargeted immune stimulation. In 1985 and 1987, Rosenberg et al. reported that recombinant human IL-2 therapy caused dramatic shrinkage of tumors in patients with advanced disease $[136,137]$. Subsequently, numerous clinical trials were conducted with systemic IL-2 and bolus high-dose IL-2 (aldesleukin) was approved for use in cancer patients by the US FDA in 1992. Since then, IL-2 therapy has been notable for inducing durable remissions in patients with metastatic renal cell carcinoma and melanoma and for significant immune-mediated toxicity [138-141]. Coincident with beneficial immunomodulatory effects, namely increased proliferation and cytokine production by $\mathrm{T}$ cells, B cells, NK cells and macrophages [139], systemic IL-2 frequently produces decreased neutrophil chemotaxis [142-144] and increased eosinophil levels $[145,146]$. Impaired neutrophil migration may increase the infection rate in patients treated with high-dose IL-2 [142-144], while eosinophilia 
may explain reports of allergic asthma and dermatitis in some patients $[139,147]$. A life-threatening side effect often observed with systemic IL-2 therapy is a syndrome resembling septic shock that entails fever, malaise and capillary leak syndrome produced by widespread activation and cytokine production (TNF- $\alpha$, IL-6, IL-1, IL-12 and GM-CSF) by macrophages and other immune effectors [139,148-151]. Recognition of the mechanisms behind systemic high-dose IL-2 toxicity has led to the development of alternative strategies, including low-dose [152], subcutaneous [153], inhaled [154], structurally modified [155] and tumor-targeted [156] IL-2 immunotherapy. Interestingly, while low-dose systemic IL-2 has a greatly reduced toxicity profile, it also has less clinical efficacy for cancer immunotherapy $[152,153]$, suggesting that similar to antiCTLA- 4 therapy, strong immune activation is both necessary for clinical benefit and causative for immune-mediated adverse events. For this reason, it is our opinion that localized IL-2 therapies, which obtain a high tumor but a low systemic IL-2 dose, will provide the most effective and safe cancer immunotherapy. Despite the toxicity drawbacks of CTLA- 4 or IL-2 immunotherapy, these reagents have produced unusually durable responses for patients with advanced disease compared with traditional chemotherapy or surgery regimens, highlighting the potential of immunotherapy to eliminate metastatic disease and induce immunologic memory $[126,138,157]$. Another significant disadvantage of untargeted, systemic immune stimulation is that it poorly recreates the microenvironmental conditions required for the generation of specific antitumor immune responses using these reagents and may instead predispose the patient to immunodeficiency states or tolerance [158-161]. Preferable is a coordinated response limited to tumor, as is seen during naturally occurring immune responses.

In an attempt to improve tumor localization of systemic immune modulatory reagents, our laboratory developed a panel of fusion proteins with monoclonal antibodies, designed tumor necrosis therapy (TNT), that can be used to deliver and retain immunotherapy reagents in solid tumors and metastases when given by systemic infusion [162-164]. TNT antibodies target necrotic regions of tumors by binding to DNA or DNA/histone targets that are only made accessible after cell death [164]. Because these antigens are species nonspecific, TNT antibodies can target necrotic tumors in both experimental animal models and man. The TNT antibodies have a number of important characteristics that make them an ideal delivery agent for immune modulators, including their lack of binding to normal tissues, their long retention time in tumors after targeting and their ability to target necrotic regions centrally in both primary and metastatic lesions [164]. In addition, the binding of TNT antibodies to sites of necrosis where danger signals are being released, localizes their payload to active areas of immune stimulation. Because of these attributes, TNT has been used to deliver radionuclides [164], immunostimulatory molecules $[87,165,166]$ and vasopermeability agents [167] to treat experimental and human tumors. To date, over 250 patients have been treated with ${ }^{131}$ I-chTNT-3 and the results show that all types of tumors tested can be targeted specifically $[168,169]$. No uptake has been seen in normal tissues regardless of the age or condition of the patients tested. In 2003, ${ }^{131}$ I-chTNT-3 became the second radiolabeled monoclonal antibody worldwide to obtain approval. The antibody was approved for the treatment of lung cancer and is currently in clinical trials for the treatment of brain and other solid tumors.

To improve the tumor delivery of chemokines and costimulatory molecules and reduce systemic toxicity, our laboratory generated a panel of fusion proteins consisting of the tumor targeting monoclonal antibody chTNT-3 and cytokines, chemokines and costimulatory molecules previously shown to be important components of an antitumor response (FIGURE7) [9,43,87,97,110,119,165,167]. By using the same carrier for all these types of reagents, comparative data was obtained to demonstrate the potential of each reagent. Moreover, data relevant to clinical translation are provided, since the same antibody may be used for both mice and humans, owing to its universal binding abilities in all species. This is not a trivial characteristic, since few, if any antibodies, are available to target murine solid tumors in immunocompetent mice. One of these constructs is discussed below to demonstrate the improved efficacy of targeted versus untargeted fusion proteins for the immunotherapy of experimental solid tumors.

\section{chTNT-3/CD137L}

Zhang et al. [119] and others have demonstrated the antitumor effects of CD137 agonist or CD137L therapy. Zhang et al. further demonstrated that tumor-targeted CD137L had a greater retention time in vivo in the tumor microenvironment and in tumor-draining lymphoid nodes and had improved antitumor effects. Taking into account the importance of the extracellular C-terminus of CD137L for its 
bioactivity, the $\mathrm{N}$-terminus of the extracellular CD137L gene was fused to the C-terminus of the chTNT-3 heavy-chain gene to construct both chTNT-3/mCD137L and Fc-mCD137L fusion genes. Whole-body clearance studies were performed in healthy $\mathrm{BALB} / \mathrm{c}$ mice to establish the in vivo half-life of chTNT-3/ $\mathrm{mCD} 137 \mathrm{~L}$ and $\mathrm{Fc}-\mathrm{mCD} 137 \mathrm{~L}$, which was found to be 18 and $24 \mathrm{~h}$, respectively. Tissue biodistribution studies in CT26 tumor-bearing $\mathrm{BALB} / \mathrm{c}$ mice were performed to determine the relative tumor uptake of each fusion protein. The uptake of radioiodinated chTNT-3/ $\mathrm{mCD} 137 \mathrm{~L}$ per gram of tumor was significantly higher than the uptake in normal organs at $24 \mathrm{~h}$ $(3.1 \% \mathrm{ID} / \mathrm{g})$ and showed even higher retention at $48 \mathrm{~h}$ postinjection $(4.5 \% \mathrm{ID} / \mathrm{g})$. As expected, Fc-mCD137L demonstrated low tumor retention over time $(0.6 \% \mathrm{ID} / \mathrm{g}$ at $48 \mathrm{~h}$ postinjection) compared with the tumor-targeted fusion protein. Examination of the tumor-draining lymph nodes, an important site for tumor immunotherapy, revealed that chTNT-3/ $\mathrm{mCD} 137 \mathrm{~L}(1.2 \% \mathrm{ID} / \mathrm{g})$ also had slightly better uptake than $\mathrm{Fc}-\mathrm{mCD} 137 \mathrm{~L}(0.5 \% \mathrm{ID} / \mathrm{g})$ at $48 \mathrm{~h}$ postinjection. The average tumor draining lymph node retention of chTNT-3/mCD137L was still much lower than that measured in tumor since necrosis is likely to be higher in the latter.

A dosing study was also performed in CT26 tumor-bearing $\mathrm{BALB} / \mathrm{c}$ mice with different doses ranging from 1 to $100 \mu \mathrm{g} /$ dose of chTNT-3/ $\mathrm{mCD} 137 \mathrm{~L}, \mathrm{Fc}-\mathrm{mCD} 137 \mathrm{~L}$ and the anti-CD137 agonist antibody $2 \mathrm{~A}$. Both chTNT-3/mCD137L and $\mathrm{Fc}-\mathrm{mCD} 137 \mathrm{~L}$ treatment produced some tumor reduction $(30-40 \%$ at higher doses) tumor reduction. At the highest doses, however, the chTNT-3/mCD137L group showed $70 \%$ tumor reduction, whereas the $\mathrm{Fc}-\mathrm{mCD} 137 \mathrm{~L}$ treated mice achieved only $30 \%$ tumor reduction (FIgure 8). This difference indicates that at this particular dosage, the localization of CD137L in tumor is critical and therefore more effective. All $2 \mathrm{~A}$-treated groups showed $95 \%$ tumor reduction; eventually became tumor free and were free of signs of toxicity throughout the 140-day observation period. Additional studies are underway to improve the potency of antibody-CD137L fusion proteins since biological activity of CD137 depends on functional homo-trimer structures. Also, while some fusion proteins benefit from targeting (e.g., CD137) others rely upon systemic action, such as LEC and B7, which showed no change or a decrease in activity when fused to chTNT-3 (data not shown).

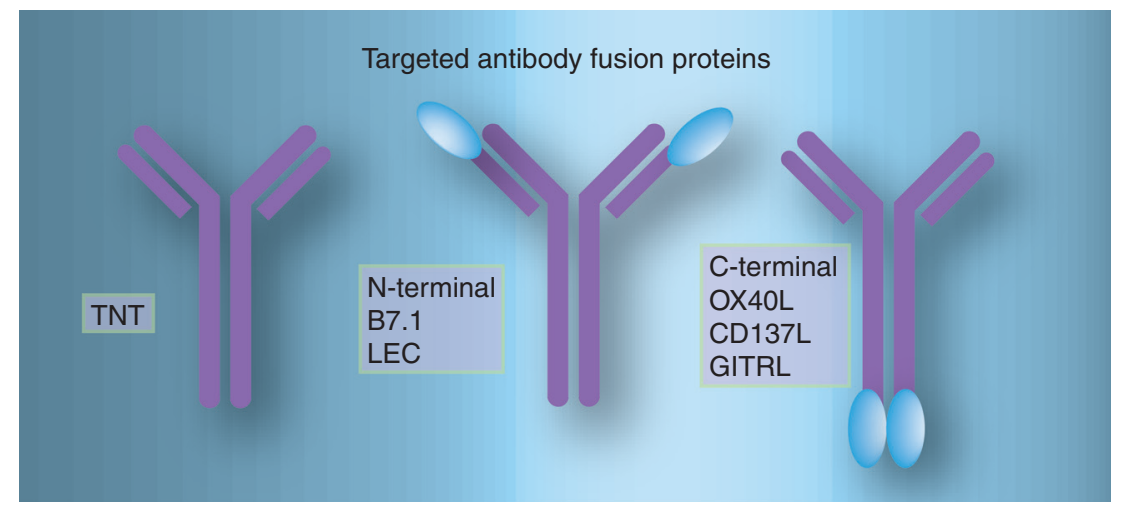

Figure 7. Tumor-targeted chemokines and costimulatory ligands using tumor necrosis treatment monoclonal antibody fusion proteins. Depending upon the location of the active site of the chemokine or costimulatory molecule, each were genetically linked to either the C- or N-terminus of the heavy chain of TNT to produce active fusion proteins. Fc-linked reagents show no specificity for tumor as demonstrated by biodistribution studies and therefore had a more systemic effect when administered intravenously than TNT fusion proteins. LEC: Liver-expressed chemokine; TNT: Tumor necrosis therapy.

\section{Combination with inhibition or depletion of immune regulatory cells}

Despite active surveillance and elimination of cancer cells by the immune system, many tumors are able to evade the immune system and therefore persist and grow to reach clinically relevant sizes. Immune suppressor cells are increasingly recognized as a major mechanism by which tumors escape immune destruction. Many currently approved immunotherapies for cancer rely upon immune stimulation, but have failed to reverse tumor immune tolerance, the second half of any effective immunotherapy and

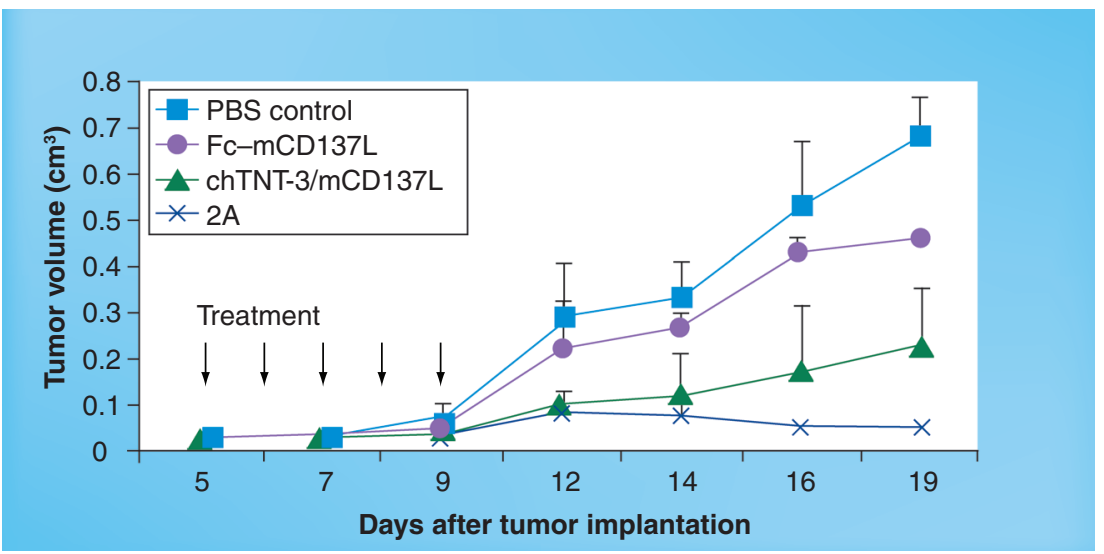

Figure 8. Tumor-targeted $m C D 137 L$ fusion protein immunotherapy shows greater antitumor efficacy compared with untargeted Fc-mCD137L in CT26 tumor-bearing mice. In these studies, groups of tumor-bearing mice $(n=5)$ received five daily doses of reagent intravenously, starting on day 5 after tumor implantation. Unlike the OX40L results shown in Figure 5, the results indicated that the agonist antibody $2 \mathrm{~A}$ was the most effective in inhibiting tumor growth in these studies. However, mice receiving chTNT-3/mCD137L showed better tumor inhibition than untargeted $\mathrm{Fc}-\mathrm{mCD} 137 \mathrm{~L}$, suggesting that that targeting this costimulatory molecule to the tumor microenvironment is more effective for treatment. PBS: Phosphate buffered saline. 


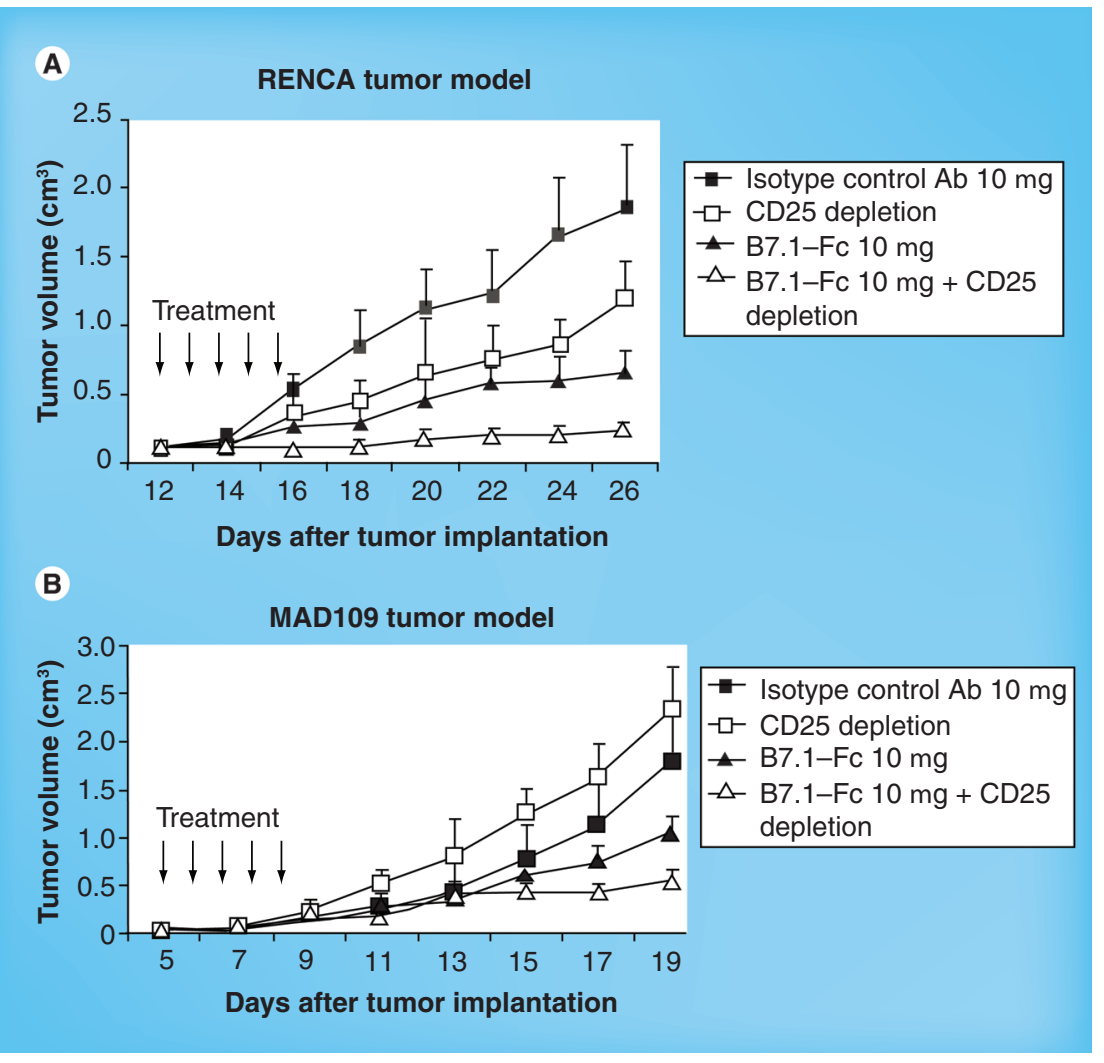

Figure 9. Effect of combination B7.1-Fc immunotherapy and CD25+ Treg depletion on the growth of RENCA and MAD109 murine solid tumors $(\mathbf{n}=\mathbf{5})$. These studies (A \& B) demonstrate that combination therapy to eliminate Tregs and provide missing costimulation with B7.1-Fc produces a more profound inhibition of tumor growth in two murine solid tumor models. Treg depletion was accomplished by administering a single $500-\mu \mathrm{g}$ ip. dose of the rat anti-CD25 monoclonal antibody PC61 on day 0.

Ab: Antibody. of CT26 colon carcinomas in mice [110]. In these studies, combined $\mathrm{Fc}-\mathrm{mGITRL}$ fusion protein and Treg depletion therapy yielded a significant improvement in the mean percentage tumor regression from $90 \%$ with Fc-mGITRL alone to $100 \%$ (complete tumor regression) with FcmGITRL and Treg depletion [78].

It is important to note that many of the pathologic ways tumors escape immune destruction are physiologic pathways, in particular induction of tolerance to self-tissues and the maternal-fetal relationship, that have been co-opted [4]. For this reason, reversal of immune tolerance for cancer therapy carries a not insignificant risk of inducing unwanted autoimmune reactions. Optimal immunotherapy then, can be said to include immune stimulation, reversal of tumor immune tolerance and specificity for the tumor. Our laboratory has paired tumor-targeted immune stimulatory immunotherapy protocols with inhibition of suppressor cells to generate robust antitumor immunity and rejection of established tumors in mice [48]. Shown in Figure 10, combination therapy with tumor-targeted chemokine CCL16 (LECchTNT-3) and anti-CD $4^{+}$or $\mathrm{CD} 25^{+}$monoclonal antibodies for Treg depletion yielded complete regression of established CT26 tumors in all experimental animals. Other studies in our laboratory and by others $[4,170,171]$ show that suppressor cell inhibition also improves the efficacy of cancer vaccines, another form of tumor-specific immune stimulation being explored for cancer immunotherapy. In addition, Tregs are only one of several immune suppressor cell populations that are recruited to the tumor microenvironment and impede immunotherapy protocols. MDSCs have only recently been recognized and characterized in cancer patients and experimental tumor models. Strategies for the inhibition of MDSCs are being developed, including selective depletion of MDSCs (5-fluorouracil, gemcitabine, docetaxel or sunitinib), inhibition of signaling pathways (sunitinib, GW2580, amiloride or HIF1 $\alpha$ inhibitors), or inhibition of suppressive mechanisms (celecoxib, sildenafil or all-trans retinoic acid) [15]. Studies of human MDSCs lag behind those of murine MDSCs, but recent studies by our laboratory have helped to define their induction, phenotype, function and transcriptional regulation to identify therapeutic targets $[69,172]$.

\section{Conclusion}

Considerable data now support the ability of immunotherapy to improve host antitumor immune responses and control tumor growth. Furthermore, successful immunotherapy trials in 
experimental models show that complete regression of well-established tumors is possible and that immunologic memory generated by robust immunity can protect the host from subsequent rechallenge by tumor cells. As such, immunotherapy holds great promise as an added clinical tool in the treatment of cancer patients. Chemokines and costimulatory molecules are two types of immune stimulatory therapy that have been shown to improve tumor infiltration by immune cells and APC-T-cell antigen priming and effector functions, respectively. When paired with mechanisms for tumor targeting and the reversal of tumor-mediated immune tolerance these immunotherapies show even greater efficacy in rejecting established tumors and promoting sustained immunity.

Optimal immunotherapy is likely to require both immune activation and reversal of tumor immune tolerance [69]. As depicted schematically in Figure 11, tumor immune escape is frequently accompanied by an absence of activated APC and effector cells (e.g., CTLs and NK cells) and the accumulation of tumor-associated suppressor cells. Failure to comprehensively address the immune dysfunction present in cancer patients will lead to disappointing clinical results and unrealized potential of these therapies. Furthermore, to reduce collateral immune effects, immunotherapy should incorporate strategies for targeting the tumor. In this way, immunotherapy can be used to enhance the induction of infiltration signals (adhesion molecules) on the vascular gateway, facilitate the expansion and licensing of cytotoxic lymphoid cells, induce the cross-presentation of tumor antigens by APCs and finally, induce a memory response required to insure a lasting and clinical outcome.

\section{Future perspective}

Cancer immunotherapy is just beginning to capture the hope and imagination of clinical
A

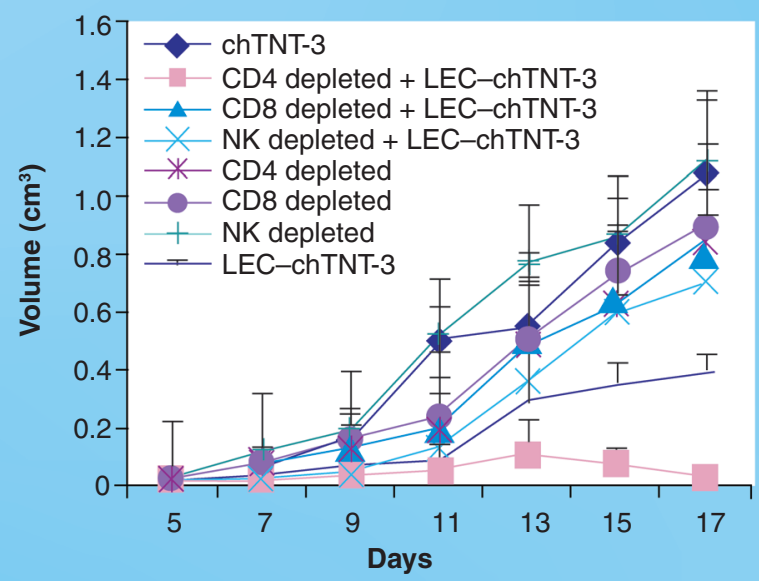

B

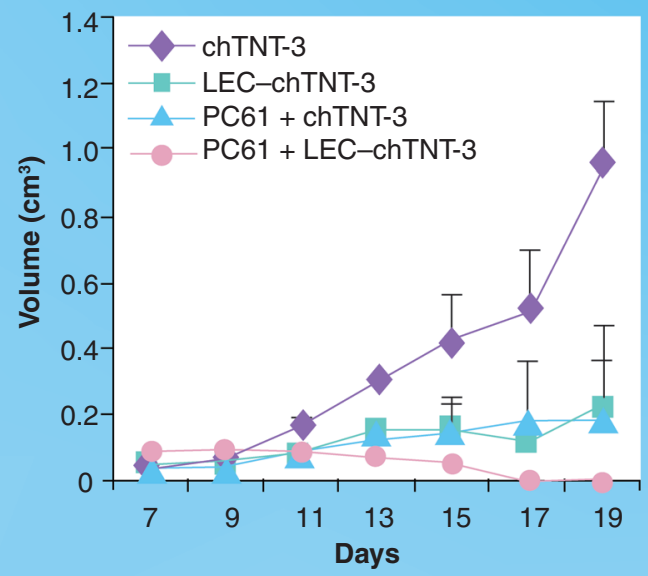

C

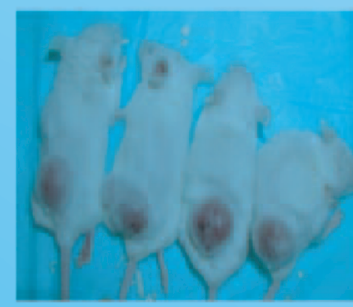

chTNT-3 control

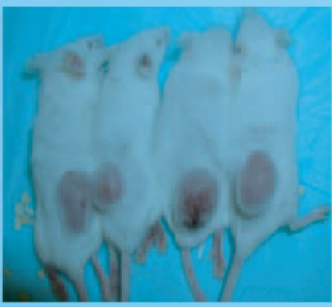

CD4 depletion

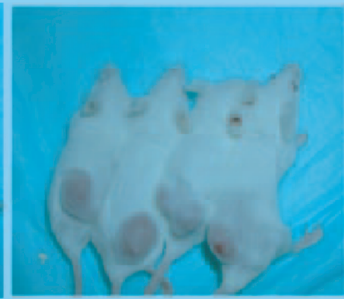

LEC-chTNT-3

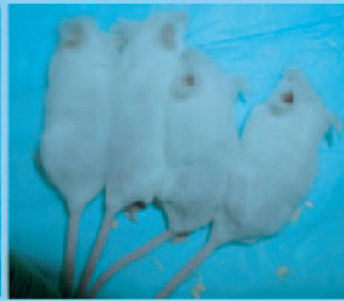

LEC-chTNT-3 + CD4 depletion

Figure 10. Complete regression of established CT26 tumors by combination LEC-chTNT-3 and Treg depletion

immunotherapy. Depletion studies with cytotoxic rat antimouse lymphoid antisera demonstrated that removal of CD8 ${ }^{+}$and NK cells prior to LEC immunotherapy prevented active tumor inhibition. By contrast, depletion of either (A) CD4+ T helper/suppressors cells or (B) CD25+ Tregs produced complete tumor regression when used in combination with LEC-chTNT-3 immunotherapy. In these experiments, the depleting rat antimouse antibodies were administered ip. as a single 500- $\mu \mathrm{g}$ dose on day 0 . Groups of mice $(n=5)$ were then measured for tumor growth by caliper measurement three times per week. As shown in (C), combination immunotherapy with tumor-targeted chemokine CCL16 (LEC-chTNT-3) and anti-CD4+ or anti-CD25+ monoclonal antibodies for Treg cell depletion produced complete regression of established tumors in all experimental animals. In comparison, monotherapy with either LEC-chTNT-3 or Treg cell depletion yielded significant reduction in tumor growth relative to untreated animals but no complete tumor cures.

LEC: Liver-expressed chemokine; TNT: Tumor necrosis therapy. 


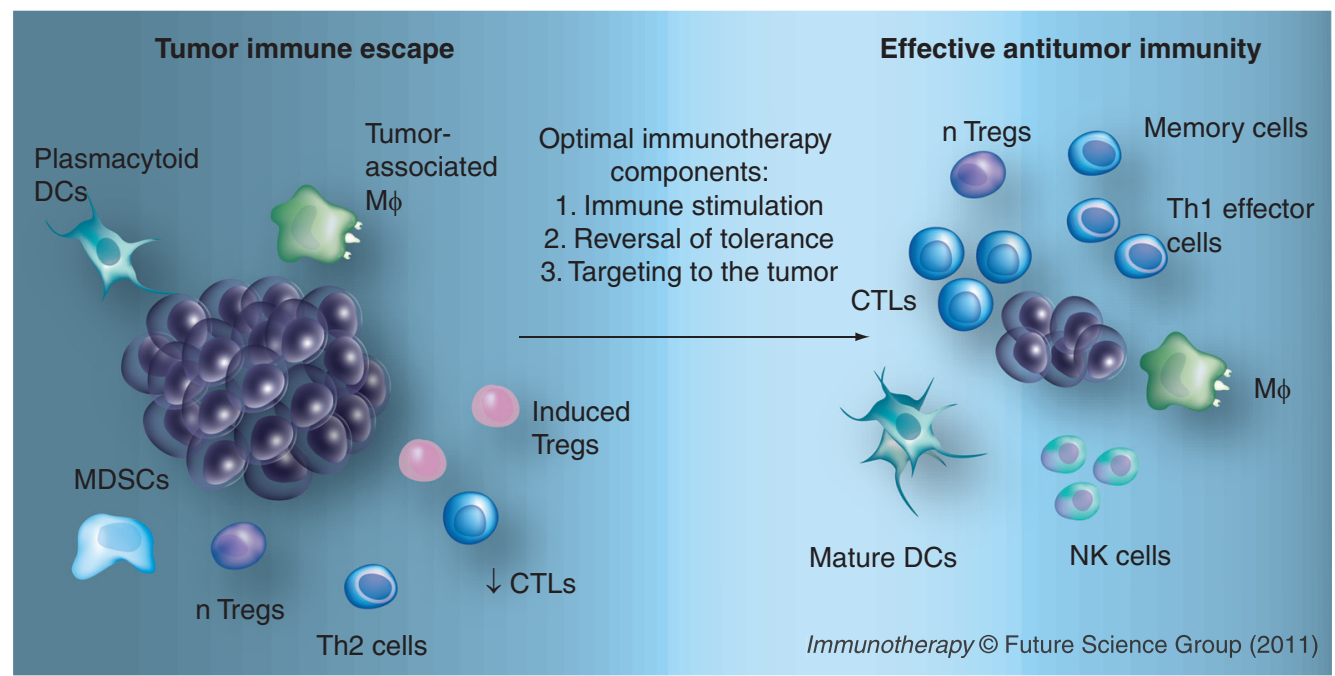

Figure 11. Optimal cancer immunotherapy combines targeted immune stimulation and reversal of tumor immune tolerance. Tumor immune surveillance mechanisms, including antigen-presenting cells (DCs, M $\phi$ and effector cells [Th cells, CTLs and NK cells]), recognize and attack neoplastic cells in the normal host to control tumor growth. When tumor immune escape occurs, immune suppressor cells recruited to the tumor microenvironment and secondary lymphoid tissues inhibit cell-mediated immunity to facilitate tumor progression. Immune suppressor cells that accumulate in the tumor setting include derivatives of innate (MDSCs, plasmacytoid DCs and tumor-associated $\mathrm{M} \phi$ ) and adaptive, or induced, Tregs. In addition to the naturally occurring Treg populations that inhibit reactions to self-antigens at steady state, induced Tregs arise in the periphery in the setting of chronic inflammation and cancer. Finally, there is a major shift in immune cytokines from cell-mediated promoting (Th1) cytokines (IFN- $\gamma$, TNF- $\alpha$ and IL-2) to humoral immunity promoting (Th2) cytokines (IL-4, IL-5 and IL-10) in the cancer setting. Immune stimulatory treatments, such as chemokines and costimulatory molecule agonists or ligands, increase tumor infiltrating leukocytes and antigen priming of effector cells. Spatial targeting of immunotherapy to the tumor microenvironment and specificity for tumor-associated antigens can decrease the risk of autoimmune reactions, increase retention time in the tumor and improve the efficacy of treatment.

CTL: Cytotoxic T lymphocyte; DC: Dendritic cell; M $\phi$ : Macrophage; MDSC: Myeloid-derived suppressor cell; Th: T helper cell.

investigators as a viable form of cancer treatment, since it holds the promise of harnessing the immune system and its memory response to insure lasting cures. As critical pieces of tumor immunology become uncovered, an integrated approach to cancer immunotherapy becomes a real possibility. In this article, several of these critical pieces are described and will undoubtedly become cornerstones for successful immunotherapy protocols in the future. Specifically, targeted reagents that focus immune modulators inside the tumor microenvironment are critical for converting immune tolerance. These immune modulators which include innate immune activators, chemokines and costimulatory molecules, have now been shown to have potent antitumor activity. However, when used with methods to delete or inhibit immunosuppression, these treatments have been found to produce lasting cures in several well-characterized murine solid tumor models. Along with tumor vaccines to provide active immune stimulation, tumor immunomodulation and inhibition of immunosuppression can now form the basis of a successful cancer immunotherapy regimen.
The challenge is now to uncover which human tumors are likely to respond to specific combination therapies, so that this integrated approach can be translated to man. Testing these concepts in murine tumor models can provide important clues, especially if their detailed characterization can be shown to be similar to what is seen in the clinic. Finally, collaboration among investigators may be needed to provide specific pieces of the immunotherapy puzzle required to test combination immunotherapy approaches, which in itself, may be the greatest challenge confronting the implementation of current scientific advances.

Financial \& competing interests disclosure

AL Epstein is a co-founder of Cancer Therapeutics Laboratories, Inc. (Los Angeles, CA). All other authors declare that they have no conflicts of interest. The authors have no other relevant affiliations or financial involvement with any organization or entity with a financial interest in or financial conflict with the subject matter or materials discussed in the manuscript apart from those disclosed.

No writing assistance was utilized in the production of this manuscript. 


\section{Executive summary}

- Immunotherapy seeks to use a patient's own immune system to eliminate cancer cells by overcoming tumor-mediated immune dysfunction and activating cell-mediated immune responses. This approach has significant advantages over conventional treatments (surgery, radiation therapy and chemotherapy) for the elimination of metastatic and recurrent disease, including systemic trafficking of immune cells, the inherent specificity of the adaptive immune system and immunologic memory for tumor antigens.

- Chemokine networks regulate physiologic cell migration that may be disrupted to inhibit antitumor immune responses or co-opted to promote tumor growth and metastasis in cancer. Recent studies highlight the potential use of chemokines in cancer immunotherapy to improve innate and adaptive cell interactions (e.g., CCL19 and CCL21) and to recruit immune effector cells into the tumor microenvironment (e.g., CCL2, CCL3, CCL5 and CCL16).

- Reciprocal expression and binding of costimulatory molecules and their ligands (e.g., CD28-B7, OX40-OX40L, GITR-GITRL and CD137-CD137L) by antigen-presenting cells and naive lymphocytes is a common immune deficit observed in cancer patients and experimental tumor models. These interactions are needed to generate robust antitumor immune responses in vivo.

- Immunotherapy approaches to replace costimulatory molecules using soluble fusion proteins, agonist antibodies, or viral-mediated expression by tumor cells significantly inhibit tumor growth and boost T-cell-mediated immunity. Therapy with costimulatory molecules is more effective when combined with endogenous antigen priming (e.g., radiation therapy and chemotherapy), tumor vaccines and cytokine therapies.

- Tumors escape host immune defenses not only by evasion (e.g., downregulation of antigen presentation and costimulatory molecules) but also by the recruitment and activation of suppressor-cell populations (e.g., Tregs, myeloid-derived suppressor cells and tumor-associated macrophages). Targeted inhibition of these cells improves the antitumor responses generated by immune modulatory ligand and chemokine immunotherapy.

- Nonspecific immune activation or reversal of tolerance carries a risk for induction of immune related adverse events, as seen in patients receiving systemic IL-2 and anti-CTLA-4 therapies. Tumor necrosis-targeted fusion proteins may provide a better level of safety owing to their preferential binding to tumors compared with untargeted reagents.

- Despite the toxicity drawbacks of CTLA-4 or IL-2 immunotherapy, these reagents have produced unusually durable responses for patients with advanced disease compared with traditional chemotherapy or surgery regimens, highlighting the potential of immunotherapy to eliminate metastatic disease and induce immunologic memory.

- Successful immunotherapy will require both advanced methods of immunostimulation and the removal of immunosuppression in the tumor and associated lymphoid tissues.

\section{Bibliography}

Papers of special note have been highlighted as: " of interest

1 Lizée G, Cantu MA, Hwu P. Less yin, more yang: confronting the barriers to cancer immunotherapy. Clin. Cancer Res. 13(18 Pt 1), 5250-5255 (2007).

2 Stewart TJ, Abrams SI. How tumours escape mass destruction. Oncogene 27, 5894-5903 (2008).

- Excellent primer on tumor immune escape for clinicians and basic scientists, written by an expert in the field of immunotherapy.

3 Stewart TJ, Smyth MJ. Improving cancer immunotherapy by targeting tumor-induced immune suppression. Cancer Metast. Rev. 30(1), 125-140 (2011).

4 Sadun RE, Sachsman SM, Chen X et al. Immune signatures of murine and human cancers reveal unique mechanisms of tumor escape and new targets for cancer immunotherapy. Clin. Cancer Res. 13, 4016-4025 (2007).

- Ground-breaking publication showing how identification of the major mechanisms of immune escape in colorectal and breast cancer can be used to tailor successful immunotherapy approaches.
5 Goldman B, DeFrancesco L. The cancer vaccine roller coaster. Nat. Biotechnol. 27(2), 129-139 (2009).

6 Palena C, Schlom J. Vaccines against human carcinomas: strategies to improve antitumor immune responses. J. Biomed. Biotechnol. 2010, 380697 (2010).

7 Eshhar Z. Adoptive cancer immunotherapy using genetically engineered designer T-cells: first steps into the clinic. Curr. Opin Mol. Ther. 12(1), 55-63 (2010).

8 Dubensky TW Jr, Reed SG. Adjuvants for cancer vaccines. Semin. Immunol. 22(3), 155-161 (2010).

9 Khawli LA, Hu P, Epstein AL. Cytokine, chemokine, and co-stimulatory fusion proteins for the immunotherapy of solid tumors. Handb. Exp. Pharmacol. 181, 291-328 (2008).

10 Katz JB, Muller AJ, Prendergast GC. Indoleamine 2,3-dioxygenase in T-cell tolerance and tumoral immune escape. Immunol. Rev. 222, 206-221 (2008).

11 Homey B, Müller A, Zlotnik A. Chemokines. Agents for the immunotherapy of cancer? Nat. Rev. Immunol. 2(3), 175-184 (2002).

- Comprehensive review of chemokines in the tumor microenvironment and progress in modulating these molecules for the treatment of cancer.
12 Placke T, Kopp HG, Salih HR. Glucocorticoid-induced TNFR-related (GITR) protein and its ligand in antitumor immunity: functional role and therapeutic modulation. Clin. Dev. Immunol. 2010, 239083 (2010).

13 Lynch DH. The promise of 4-1BB (CD137)-mediated immunomodulation and the immunotherapy of cancer. Immunol. Rev. 222, 277-286 (2008).

14 Jensen SM, Maston LD, Gough MJ et al. Signaling through OX40 enhances antitumor immunity. Semin. Oncol. 37(5), 524-532 (2010).

15 Lechner MG, Epstein AL. A new mechanism for blocking myeloid-derived suppressor cells by CpG. Clin. Cancer Res. 17(7), 1645-1648 (2011).

16 Stewart TJ, Smyth MJ. Chemokine - chemokine receptors in cancer immunotherapy. Immunotherapy 1(1), 109-127 (2009).

17 Slettenaar VI, Wilson JL. The chemokine network: a target in cancer biology? Adv. Drug Deliv. Rev. 58(8), 962-974 (2006).

18 Mantovani A, Savino B, Locati M, Zammataro L, Allavena P, Bonecchi R. The chemokine system in cancer biology and therapy. Cytokine Growth Factor Rev. 21(1), 27-39 (2010). 
19 Balkwill F. Cancer and the chemokine network. Nat. Rev. Cancer 4, 540-550 (2004).

20 Rossi D, Zlotnik A. The biology of chemokines and their receptors. Annu. Rev. Immunol. 18, 217-242 (2000).

21 Coussens LM, Werb Z. Inflammation and cancer. Nature 420(6917), 860-867 (2002).

22 Belperio JA, Keane MP, Arenberg DA et al. CXC chemokines in angiogenesis. J. Leukoc. Biol. 68(1), 1-8 (2000).

23 Förster R, Schubel A, Breitfeld D et al. CCR7 coordinates the primary immune response by establishing functional microenvironments in secondary lymphoid organs. Cell 99, 23-33 (1999).

24 Sharma S, Stolina M, Luo J et al. Secondary lymphoid tissue chemokine mediates $\mathrm{T}$ cell-dependent antitumor responses in vivo. J. Immunol. 164, 4558-4563 (2000).

25 Vicari AP, Ait-Yahia S, Chemin K, Mueller A, Zlotnik A, Caux C. Antitumor effects of the mouse chemokine 6Ckine/SLC through angiostatic and immunological mechanisms. J. Immunol. 165(4), 1992-2000 (2000).

26 Braun SE, Chen K, Foster RG et al. CC chemokine CK $\beta-11 /$ MIP-3 $\beta$ /ELC/Exodus 3 mediates tumor rejection of murine breast cancer cells through NK cells. J. Immunol. 164, 3200-3206 (2000).

27 Kirk CJ, Hartigan-O'Connor D, Nickoloff BJ et al. $\mathrm{T}$ cell-dependent antitumor immunity mediated by secondary lymphoid tissue chemokine: augmentation of dendritic cell-based immunotherapy. Cancer Res. 61(5), 2062-2070 (2001).

28 Kirk CJ, Hartigan-O’Connor D, Mulé JJ. The dynamics of the T-cell antitumor response: chemokine-secreting dendritic cells can prime tumor-reactive $\mathrm{T}$ cells extranodally. Cancer Res. 61(24), 8794-8802 (2001).

29 Shields JD, Kourtis IC, Tomei AA, Roberts JM, Swartz MA. Induction of lymphoid-like stroma and immune escape by tumors that express the chemokine CCL21. Science 328(5979), 749-752 (2010).

30 Dubinett SM, Lee JM, Sharma S, Mulé JJ. Chemokines: can effector cells be redirected to the site of the tumor? Cancer J. 16(4), 325-335 (2010).

31 Baratelli F, Takedatsu H, Hazra S et al. Pre-clinical characterization of GMP grade CCL21-gene modified dendritic cells for application in a Phase I trial in non-small cell lung cancer. J. Trans. Med. 6, 38 (2008).

32 Kar UK, Srivastava MK, Andersson A et al. Novel. CCL21-nault vanocapsule intratumoral delivery inhibits lung cancer growth. PLoS ONE 6(5), E18758 (2011).
33 Fang L, Hwang ST. Roles for CCR7 in cancer biology. In: Chemokines Receptors in Cancer, Cancer Drug Discovery and Development. Fulton AM (Ed.). Humana Press, NY, USA 93-108 (2009).

34 Ben-Baruch A. Organ selectivity in metastasis: regulation by chemokines and their receptors. Clin. Exp. Metast. 25(4), 345-356 (2008).

35 Wiley HE, Gonzalez EB, Maki W, Wu MT, Hwang ST. Expression of CC chemokine receptor-7 and regional lymph node metastasis of B16 murine melanoma. J. Natl Cancer Inst. 93(21), 1638-1643 (2001).

36 Fang L, Lee VC, Cha E, Zhang H, Hwang ST. CCR7 regulates B16 murine melanoma cell tumorigenesis in skin. J. Leukoc. Biol. 84(4), 965-972 (2008).

37 Muller A, Homey B, Soto $\mathrm{H}$ et al. Involvement of chemokine receptors in breast cancer metastasis. Nature 410, 50-56 (2001).

38 Wilson JL, Burchell J, Grimshaw MJ. Endothelins induce CCR7 expression by breast tumor cells via endothelin receptor A and hypoxia-inducible factor-1. Cancer Res. 66(24), 11802-11807 (2006).

39 Buonamici S, Trimarchi T, Ruocco MG et al. CCR7 signalling as an essential regulator of CNS infiltration in T-cell leukaemia. Nature 459(7249), 1000-1004 (2009).

40 Cappello P, Fraone T, Barberis L et al. CC-chemokine ligand 16 induces a novel maturation program in human immature monocyte-derived dendritic cells. J. Immunol. 177(9), 6143-6151 (2006).

41 Howard OMZ, Dong HF, Shirakawa AK, Oppenheim JJ. LEC induces chemotaxis and adhension by interacting with CCR 1 and CCR8. Blood 96, 840-845 (2000).

42 Giovarelli M, Cappello P, Forni G et al. Tumor rejection and immune memory elicited by locally released LEC chemokine are associated with and impressive recruitment of APCs, lymphocytes, and granulocytes. J. Immunol. 164, 3200-3206 (2000).

43 Li J, Hu P, Khawli LA, Epstein AL. Complete regression of experimental solid tumors by combination LEC-chTNT-3 immunotherapy and CD25(+) T-cell depletion. Cancer Res. 63(23), 8384-8392 (2003).

- Demonstrates effective immunotherapy of established tumors using an immunotherapy approach that combines tumor-targeted immune stimulation with removal of immunosuppression in the host.

44 Lapteva N, Huang XF. CCL5 as an adjuvant for cancer immunotherapy. Exp. Opin. Biol. Ther. 10(5), 725-733 (2010).
45 Menten P, Wuyts A, Van Damme J. Macrophage inflammatory protein-1. Cytokine Growth Factor Rev. 13(6), 455-481 (2002).

46 Soria G, Ben-Baruch A. The inflammatory chemokines CCL2 and CCL5 in breast cancer. Cancer Lett. 267(2), 271-285 (2008).

47 Chen D, Bromberg JS. T regulatory cells and migration. Am. J. Trans. 6(7), 1518-1523 (2006).

48 Murdoch C, Muthana M, Coffelt SB, Lewis CE. The role of myeloid cells in the promotion of tumour angiogenesis. Nat. Rev. Cancer 8(8), 618-631 (2008).

49 Fridlender ZG, Buchlis G, Kapoor V et al. CCL2 blockade augments cancer immunotherapy. Cancer Res. 70 (1), 109-118 (2010).

50 Zhu X, Fujita M, Snyder LA, Okada H. Systemic delivery of neutralizing antibody targeting CCL2 for glioma therapy. J. Neurooncol. 104(1), 83-92 (2010).

51 Zhang J, Lu Y, Pienta KJ. Multiple roles of chemokine (C-C motif) ligand 2 in promoting prostate cancer growth. J. Natl. Cancer Inst. 102(8), 522-528 (2010).

52 Parker JN, Meleth S, Hughes KB, Gillespie GY, Whitley RJ, Markert JM. Enhanced inhibition of syngeneic murine tumors by combinatorial therapy with genetically engineered HSV-1 expressing CCL2 and IL-12. Cancer Gene Ther. 12(4), 359-368 (2005).

53 Nagai M, Masuzawa T. Vaccination with MCP-1 cDNA transfectant on human malignant glioma in nude mice induces migration of monocytes and NK cells to the tumor. Int. Immunopharmacol. 1(4), 657-664 (2001).

54 Hirose K, Hakozaki M, Nyunoya Y et al. Chemokine gene transfection into tumour cells reduced tumorigenicity in nude mice in association with neutrophilic infiltration. Br. J. Cancer 72(3), 708-714 (1995).

55 Cao Q, Jin Y, Jin M et al. Therapeutic effect of MIP-1 $\alpha$-recruited dendritic cells on preestablished solid and metastatic tumors. Cancer Lett. 295(1), 17-26 (2010).

56 Aravindaram K, Yu HH, Lan CW et al. Transgenic expression of human gp100 and RANTES at specific time points for suppression of melanoma. Gene Ther. 16(11), 1329-1339 (2009).

57 Inoue $\mathrm{H}$, Iga $\mathrm{M}$, Xin $\mathrm{M}$ et al. TARC and RANTES enhance antitumor immunity induced by the GM-CSF-transduced tumor vaccine in a mouse tumor model. Cancer Immunol. Immunother. 57(9), 1399-1411 (2008). 
58 Song S, Liu C, Wang J et al. Vaccination with combination of Fit3L and RANTES in a DNA prime-protein boost regimen elicits strong cell-mediated immunity and antitumor effect. Vaccine 27(7), 1111-1118 (2009).

59 Maksym RB, Tarnowski M, Grymula Ket al. The role of stromal-derived factor-1-CXCR7 axis in development and cancer.

Eur. J. Pharmacol. 625(1-3), 31-40 (2009).

60 Karin N. The multiple faces of CXCL12 $($ SDF-1 $\alpha)$ in the regulation of immunity during health and disease. J. Leukoc. Biol. 88(3), 463-473 (2010).

61 Sun X, Cheng G, Hao M et al. CXCL12/CXCR4/CXCR7 chemokine axis and cancer progression. Cancer Metast. Rev. 29(4), 709-722 (2010).

- Review of CXCL12 and its cognate receptors that elegantly presents the duality of chemokines in the tumor-bearing host as both tumor promoting and inhibiting.

62 Teicher BA, Fricker SP. CXCL12 (SDF-1)/ CXCR4 pathway in cancer. Clin. Cancer Res. 16(11), 2927-2931 (2010).

63 Zou W, Machelon V, Coulomb-L'Hermin A et al. Stromal-derived factor-1 in human tumors recruits and alters the function of plasmacytoid precursor dendritic cells. Nat. Med. 7(12), 1339-1346 (2001).

64 Sun YX, Schneider A, Jung Y et al. Skeletal localization and neutralization of the SDF-1(CXCL12)/CXCR4 axis blocks prostate cancer metastasis and growth in osseous sites in vivo. J. Bone Miner. Res. 20 (2), 318-329 (2005).

65 Liang Z, Yoon Y, Votaw J, Goodman MM, Williams L, Shim H. Silencing of CXCR4 blocks breast cancer metastasis. Cancer Res. 65(3), 967-971 (2005).

66 Miao Z, Luker KE, Summers BC et al. CXCR7 (RDC1) promotes breast and lung tumor growth in vivo and is expressed on tumor-associated vasculature. Proc. Natl Acad. Sci. USA 104(40), 15735-15740 (2007).

67 Salmaggi A, Maderna E, Calatozzolo C et al. CXCL12, CXCR4 and CXCR7 expression in brain metastases. Cancer Biol. Ther. 8(17), 1608-1614 (2009).

68 Lee CH, Kakinuma T, Wang J et al. Sensitization of B16 tumor cells with a CXCR4 antagonist increases the efficacy of immunotherapy for established lung metastases. Mol. Cancer Ther. 5(10), 2592-2599 (2006).

69 Lechner MG, Megiel C, Russell S et al. Functional characterization of human CD33+ and $\mathrm{CD} 11 \mathrm{~b}^{+}$myeloid-derived suppressor cell subsets induced by a diverse set of human solid tumor cell lines. J. Transl Med. 9, 90 (2011).
70 Corzo CA, Condamine T, Lu L et al. HIF- $1 \alpha$ regulates function and differentiation of myeloid-derived suppressor cells in the tumor microenvironment. J. Exp. Med. 207(11), 2439-2453 (2010).

71 Eltzschig KR, Carmeliet P. Hypoxia and inflammation. N. Engl. J. Med. 364(7), 656-665 (2011).

72 Doedens AL, Stockmann C, Rubinstein MP et al. Macrophage expression of hypoxiainducible factor- $1 \alpha$ suppresses T-cell function and promotes tumor progression. Cancer Res. 70 (19), 7465-7475 (2010).

73 Semenza GL. Targeting HIF-1 for cancer therapy. Nat. Rev. Cancer. 3(10), 721-732 (2003).

74 Steinberg M, Silva M. Plerixafor. A chemokine receptor- 4 antagonist for mobilization of hematopoietic stem cells for transplantation after high-dose chemotherapy for non-Hodgkin's lymphoma or multiple myeloma. Clin. Ther. 32(5), 821-843 (2010).

75 Hartmann TN, Grabovsky V, Pasvolsky R et al. A crosstalk between intracellular CXCR7 and CXCR4 involved in rapid CXCL12-triggered integrin activation but not in chemokine-triggered motility of human T lymphocytes and CD34+ cells. J. Leukoc. Biol. 84(4), 1130-1140 (2008).

76 Hathcock KS, Laszlo G, Pucillo C, Linsley P, Hodes RJ. Comparative analysis of B7-1 and B7-2 co-stimulatory ligands: expression and function. J. Exp. Med. 180, 631-640 (1994).

77 Abken H, Hombach A, Heuser C, Kronfeld K, Seliger B. Tuning tumor-specific T-cell activation: a matter of costimulation? Trends Immunol. 23(5), 240-245 (2002).

78 Khawli LA, Hu P, Epstein AL. Targeted and untargeted fusion proteins: current approaches to cancer immunotherapy. In: Fusion Protein Technologies for Biopharmaceuticals: Applications and Challenges. Wiley-Blackwell Press (2011) (In Press).

79 Townsend SE, Allison JP. Tumor rejection after direct costimulation of $\mathrm{CD} 8^{+} \mathrm{T}$ cells by B7-transfected melanoma cells. Science 259(5093), 368-370 (1993).

80 Chong H, Todryk S, Hutchinson G, Hart IR, Vile RG. Tumour cell expression of B7 costimulatory molecules and interleukin-12 or granulocyte-macrophage colony-stimulating factor induces a local antitumour response and may generate systemic protective immunity. Gene Ther. 5(2), 223-232 (1998).

81 Hurwitz AA, Townsend SE, Yu TF, Wallin JA, Allison JP. Enhancement of the anti-tumor immune response using a combination of interferon- $\gamma$ and B7 expression in an experimental mammary carcinoma. Int. J. Cancer. 77(1), 107-113 (1998).
82 Palena C, Foon KA, Panicali D et al. Potential approach to immunotherapy of chronic lymphocytic leukemia (CLL): enhanced immunogenicity of CLL cells via infection with vectors encoding for multiple costimulatory molecules. Blood 106(10), 3515-3523 (2005).

83 Kaufman HL, Cohen S, Cheung K et al. Local delivery of vaccinia virus expressing multiple costimulatory molecules for the treatment of established tumors. Hum. Gene Ther. 17(2), 239-244 (2006).

84 Yamaguchi N, Hiraoka SI, Mukai T et al. Induction of tumor regression by administration of B7-Ig fusion proteins: mediation by type $2 \mathrm{CD} 8^{+} \mathrm{T}$-cells and dependence on IL-4 production. J. Immunol. 172, 1347-1354 (2004).

85 Sturmhoefel K, Lee K, Gray GS et al. Potent activity of soluble B7-IgG fusion proteins in therapy of established tumors and as vaccine adjuvant. Cancer Res. 59(19), 4964-4972 (1999).

86 Liu A, Hu P, Khawli LA, Epstein AL. Combination B7-Fc fusion protein treatment and Treg cell depletion therapy. Clin. Cancer Res. 11(23), 8492-8502 (2005).

87 McHugh RS, Nagarajan S, Wang YC, Sell KW, Selvaraj P. Protein transfer of glycosylphosphatidylinositol-B7-1 into tumor cell membranes: a novel approach to tumor immunotherapy. Cancer Res. 59, 2433-2437 (1999).

88 Chen L, McGowan P, Ashe S et al. Tumor immunogenicity determines the effect of B7 costimulation on T cell-mediated tumor immunity. J. Exp. Med. 179(2), 523-532 (1994).

89 Lohr F, Hu K, Haroon Z et al. Combination treatment of murine tumors by adenovirusmediated local B7/IL12 immunotherapy and radiotherapy. Mol. Ther. 2(3), 195-203 (2000).

90 Aarts WM, Schlom J, Hodge JW. Vector-based vaccine/cytokine combination therapy to enhance induction of immune responses to a self-antigen and antitumor activity. Cancer Res. 62 (20), 5770-5777 (2002).

91 Runyon K, Lee K, Zuberek K, Collins M, Leonard JP, Dunussi-Joannopoulos K. The combination of chemotherapy and systemic immunotherapy with soluble B7-immunoglobulin G leads to cure of murine leukemia and lymphoma and demonstration of tumor-specific memory responses. Blood 97(8), 2420-2426 (2001).

92 Abbas AK, Lichtman AH. Basic Immunology Updated Edition: Functions and Disorders of the Immune System (3rd Edition). Saunders, China (2011). 
93 Gough MJ, Weinberg AD. OX40 (CD134) and OX40L. Adv. Exp. Med. Biol. 647, 94-107 (2009).

94 Weinberg AD, Rivera MM, Prell R et al. Engagement of the OX-40 receptor in vivo enhances antitumor immunity. J. Immunol. 164, 2160-2169 (2000).

95 Ali SA, Ahmad M, Lynam J et al. Antitumour therapeutic efficacy of OX40L in murine tumour model. Vaccine 22(27-28), 3585-3594 (2004).

96 Morris A, Vetto JT, Ramstad T et al. Induction of anti-mammary cancer immunity by engaging the OX-40 receptor in vivo. Breast Cancer Res. Treat. 67(1), 71-80 (2001).

97 Sadun RE, Hsu WE, Zhang N et al. $\mathrm{Fc}-\mathrm{mOX} 40 \mathrm{~L}$ fusion protein produces complete remission and enhanced survival in 2 murine tumor models. J. Immunother. 31(3), 235-245 (2008).

98 Redmond WL, Gough MJ, Weinberg AD. Ligation of the OX40 co-stimulatory receptor reverses self- $\mathrm{Ag}$ and tumor-induced $\mathrm{CD} 8$ T-cell anergy in vivo. Eur. J. Immunol. 39(8), 2184-2194 (2009).

99 Kjaergaard J, Tanaka J, Kim JA, Rothchild K, Weinberg A, Shu S. Therapeutic efficacy of OX-40 receptor antibody depends on tumor immunogenicity and anatomic site of tumor growth. Cancer Res. 60 (19), 5514-5521 (2000).

- Work by leaders in the field of cancer immunotherapy with T-cell costimulatory molecules demonstrating the role of tumor immunogenicity in developing successful immunotherapy protocols.

100 Murata S, Ladle BH, Kim PS et al. OX40 costimulation synergizes with GM-CSF whole-cell vaccination to overcome established CD $8+\mathrm{T}$ cell tolerance to an endogenous tumor antigen. J. Immunol. 176(2), 974-983 (2006).

101 Yokouchi H, Yamazaki K, Chamoto Ket al. Anti-OX40 monoclonal antibody therapy in combination with radiotherapy results in therapeutic antitumor immunity to murine lung cancer. Cancer Sci. 99(2), 361-367 (2008).

102 Gough MJ, Crittenden MR, Sarff M et al. Adjuvant therapy with agonistic antibodies to CD134 (OX40) increases local control after surgical or radiation therapy of cancer in mice. J. Immunother. 33(8), 798-809 (2010).

103 Hirschhorn-Cymerman D, Rizzuto GA, Merghoub T et al. OX40 engagement and chemotherapy combination provides potent antitumor immunity with concomitant regulatory T cell apoptosis. J. Exp. Med. 206(5), 1103-1116 (2009).
104 Nocentini G, Giunchi L, Ronchetti S et al. A new member of the tumor necrosis factor/nerve growth factor receptor family inhibits T cell receptor-induced apoptosis. Proc. Natl Acad. Sci. USA 94(12), 6216-6221 (1997).

105 Stephens GL, McHugh RS, Whitters MJ et al. Engagement of glucocorticoid-induced TNFR family-related receptor on effector $\mathrm{T}$ cells by its ligand mediates resistance to suppression by $\mathrm{CD} 4{ }^{+} \mathrm{CD} 25^{+} \mathrm{T}$ cells. J. Immunol. 173(8), 5008-5020 (2004).

106 Gurney AL, Marsters SA, Huang RM et al. Identification of a new member of the tumor necrosis factor family and its receptor, a human ortholog of mouse GITR. Curr. Biol. 9(4), 215-218 (1999).

107 Cho JS, Hsu JV, Morrison SL. Localized expression of GITR-L in the tumor microenvironment promotes $\mathrm{CD} 8^{+} \mathrm{T}$ cell dependent anti-tumor immunity. Cancer Immunol. Immunother. 58(7), 1057-1069 (2009).

108 Piao J, Kamimura Y, Iwai $\mathrm{H}$ et al. Enhancement of T-cell-mediated anti-tumour immunity via the ectopically expressed glucocorticoid-induced tumour necrosis factor receptor-related receptor ligand (GITRL) on tumours. Immunology 127(4), 489-499 (2009).

109 Nishikawa H, Kato T, Hirayama M et al. Regulatory $\mathrm{T}$ cell-resistant $\mathrm{CD} 8^{+} \mathrm{T}$ cells induced by glucocorticoid-induced tumor necrosis factor receptor signaling. Cancer Res. 68(14), 5948-5954 (2008).

$110 \mathrm{Hu}$ P, Arias RS, Sadun RE et al. Construction and preclinical characterization of Fc-mGITRL for the immunotherapy of cancer. Clin. Cancer Res. 14(2), 579-588 (2008).

111 Imai N, Ikeda H, Tawara I et al. Glucocorticoid-induced tumor necrosis factor receptor stimulation enhances the multifunctionality of adoptively transferred tumor antigen-specific CD8 ${ }^{+} \mathrm{T}$ cells with tumor regression. Cancer Sci. 100(7), 1317-1325 (2009).

112 Schwarz H, Blanco FJ, von Kempis J, Valbracht J, Lotz M. ILA, a member of the human nerve growth factor/tumor necrosis factor receptor family, regulates T-lymphocyte proliferation and survival. Blood 87(7), 2839-2845 (1996).

113 Hurtado JC, Kim YJ, Kwon BS. Signals through $4-1 \mathrm{BB}$ are costimulatory to previously activated splenic $T$ cells and inhibit activation-induced cell death. J. Immunol. 158(6), 2600-2609 (1997).

114 Takahashi C, Mittler RS, Vella AT. Cutting edge: $4-1 \mathrm{BB}$ is a bona fide CD8 T cell survival signal. J. Immunol. 162(9), 5037-5040 (1999).
115 Laderach D, Movassagh M, Johnson A, Mittler RS, Galy A. 4-1BB co-stimulation enhances human $\mathrm{CD} 8\left(^{+}\right) \mathrm{T}$ cell priming by augmenting the proliferation and survival of effector CD8 $\left(^{+}\right)$T cells. Int. Immunol. 14(10), 1155-1167 (2002).

116 Halstead ES, Mueller YM, Altman JD, Katsikis PD. In vivo stimulation of CD137 broadens primary antiviral CD81 T cell responses. Nat. Immunol. 3(6), 536-541 (2002).

117 Shuford WW, Klussman K, Tritchler DD et al. 4-1BB costimulatory signals preferentially induce $\mathrm{CD}^{+} \mathrm{T}$ cell proliferation and lead to the amplification in vivo of cytotoxic T cell responses. J. Exp. Med. 186(1), 47-55 (1997).

118 Melero I, Shuford WW, Newby SA et al. Monoclonal antibodies against the 4-1BB T-cell activation molecule eradicate established tumors. Nat. Med. 3(6), 682-685 (1997).

119 Zhang N, Sadun RE, Arias RS et al. Targeted and untargeted CD137L fusion proteins for the immunotherapy of experimental solid tumors. Clin. Cancer Res. 13(9), 2758-2767 (2007).

120 Miller RE, Jones J, Le T et al. 4-1BB-specific monoclonal antibody promotes the generation of tumor-specific immune responses by direct activation of CD8 $\mathrm{T}$ cells in a CD40dependent manner. J. Immunol. 169(4), 1792-1800 (2002).

121 Li B, Lin J, Vanroey M, Jure-Kunkel M, Jooss K. Established B16 tumors are rejected following treatment with GM-CSF-secreting tumor cell immunotherapy in combination with anti-4-1BB mAb. Clin. Immunol. 125(1), 76-87 (2007).

122 Ito F, Li Q, Shreiner AB et al. Anti-CD137 monoclonal antibody administration augments the antitumor efficacy of dendritic cell-based vaccines. Cancer Res. 64(22), 8411-8419 (2004).

123 Linsley PS, Wallace PM, Johnson J et al. Immunosuppression in vivo by a soluble form of the CTLA- $4 \mathrm{~T}$ cell activation molecule. Science 257(5071), 792-795 (1992).

124 Linsley PS, Greene JL, Tan P et al. Coexpression and functional cooperation of CTLA- 4 and CD 28 on activated T lymphocytes. J. Exp. Med. 176(6), 1595-1604 (1992).

125 Kaehler KC, Piel S, Livingstone E, Schilling B, Hauschild A, Schadendorf D. Update on immunologic therapy with anti-CTLA-4 antibodies in melanoma: identification of clinical and biological response patterns, immune-related adverse events, and their management. Semin. Oncol. 37(5), 485-498 (2010). 
- Clinically relevant review of anti-CTLA-4 therapies providing a nuanced summary of current clinical trials and suggesting the need to utilize different response criteria to evaluate these and other immunotherapies.

126 Callahan MK, Wolchok JD, Allison JP. Anti-CTLA-4 antibody therapy: immune monitoring during clinical development of a novel immunotherapy. Semin. Oncol. 37(5), 473-484 (2010).

127 Ribas A, Hauschild A, Kefford R et al. Phase III, open label, randomized, comparative study of tremelimumab (CP-675,206) and chemotherapy (temozolomide [TMZ] or dacarbazine [DTIC]) in patients with advanced melanoma. Presented at: 2008 ASCO Annual Meeting. Chicago, IL, USA, 30 May-3 June 2008.

128 Hamid O, Chin K, Li J et al. Dose effect of ipilimumab in patients with advanced melanoma: results from a Phase II, randomized, dose-ranging study. Presented at: 2008 ASCO Annual Meeting. Chicago, IL, USA, 30 May-3 June 2008.

129 O’Day SJ, Maio M, Chiarion-Sileni V et al. Efficacy and safety of ipilimumab monotherapy in patients with pretreated advanced melanoma: a multicenter single-arm Phase II study. Ann. Oncol. 21(8), 1712-1717 (2010).

130 Weber JS, Berman D, Siegel J et al. Safety and efficacy of ipilimumab with or without prophylactic budesonide in treatment-naive and previously treated patients with advanced melanoma. Presented at: 2008 ASCO Annual Meeting. Chicago, IL, USA, 30 May-3 June 2008.

131 Robert C, Thomas L, Bondarenko I et al. Ipilimumab plus dacarbazine for previously untreated metastatic melanoma. $N$. Engl. J. Med. 364 (26), 2517-2526 (2011).

132 Lynch TJ, Bondarenko IN, Luft A et al. Phase II trial of ipilimumab (IPI) and paclitaxel/carboplatin (P/C) in first-line stage IIIb/IV non-small cell lung cancer (NSCLC). Presented at: 2010 ASCO Annual Meeting. Chicago, IL, USA, 4-8 June 2010.

133 Harzstark AL, Fong L, Weinberg VKet al. Final results of a Phase I study of CTLA-4 blockade in combination with GM-CSF for metastatic castration resistant prostate cancer (mCRPC). Presented at: 2010 ASCO Annual Meeting. Chicago, IL, USA, 4-8 June 2010.

134 Weber J. Review: anti-CTLA-4 antibody ipilimumab: case studies of clinical response and immune-related adverse events. Oncologist 12(7), 864-872 (2007).

135 Di Giacomo AM, Biagioli M, Maio M. The emerging toxicity profiles of antiCTLA- 4 antibodies across clinical indications. Semin. Oncol. 37(5), 499-507 (2010).
136 Rosenberg SA, Lotze MT, Muul LM et al. Observations on the systemic administration of autologous lymphokine-activated killer cells and recombinant interleukin-2 to patients with metastatic cancer. N. Engl. J. Med. 313(23), 1485-1492 (1985).

137 Rosenberg SA, Lotze MT, Muul LM et al. A progress report on the treatment of 157 patients with advanced cancer using lymphokine-activated killer cells and interleukin-2 or high-dose interleukin-2 alone. N. Engl. J. Med. 316(15), 889-897 (1987).

138 Shablak A, Sikand K, Shanks JH, Thistlethwaite F, Spencer-Shaw A, Hawkins RE. High-dose interleukin-2 can produce a high rate of response and durable remissions in appropriately selected patients with metastatic renal cancer. J. Immunother. 34(1), 107-112 (2011).

- Highlights the durable nature of clinical responses achieved by successful immunotherapy and emphasizes that such treatment approaches may need to be tailored to the patient's specific tumor immune profile.

139 Schmidinger M, Hejna M, Zielinski CC. Aldesleukin in advanced renal cell carcinoma. Exp. Rev. Anticancer Ther. 4(6), 957-980 (2004).

140 Coppin C, Le L, Porzsolt F, Wilt T. Targeted therapy for advanced renal cell carcinoma. Cochrane Database Syst. Rev. 2, CD006017 (2008).

141 Atkins MB, Lotze MT, Dutcher JP et al. High-dose recombinant interleukin 2 therapy for patients with metastatic melanoma: analysis of 270 patients treated between 1985 and 1993. J. Clin. Oncol. 17(7), 2105-2116 (1999).

142 Jablons D, Bolton E, Mertins S et al. IL-2-based immunotherapy alters circulating neutrophil $\mathrm{Fc}_{\mathrm{c}}$ receptor expression and chemotaxis. J. Immunol. 144(9), 3630-3636 (1990).

143 Whittington R, Faulds D. Interleukin-2. A review of its pharmacological properties and therapeutic use in patients with cancer. Drugs 46(3), 446-514 (1993).

144 Klempner MS, Noring R, Mier JW, Atkins MB. An acquired chemotactic defect in neutrophils from patients receiving interleukin-2 immunotherapy. N. Engl. J. Med. 322(14), 959-965 (1990).

145 Buzio C, De Palma G, Passalacqua R et al. Effectiveness of very low doses of immunotherapy in advanced renal cell cancer. Br. J. Cancer. 76(4), 541-544 (1997).

146 Pavone L, Andrulli S, Santi R, Majori M, Buzio C. Long-term treatment with low doses of interleukin- 2 and interferon- $\alpha$ : immunological effects in advanced renal cell cancer. Cancer Immunol. Immunother. 50(2), 82-86 (2001).

147 Loppow D, Huland E, Heinzer $\mathrm{H}$ et al. Interleukin-2 inhalation therapy temporarily induces asthma-like airway inflammation. Eur. J. Med. Res. 12(11), 556-562 (2007).

148 Margolin KA, Rayner AA, Hawkins MJ et al. Interleukin-2 and lymphokine-activated killer cell therapy of solid tumors: analysis of toxicity and management guidelines. J. Clin. Oncol. 7(4), 486-498 (1989).

149 Belldegrun A, Webb DE, Austin HA 3rd et al. Effects of interleukin-2 on renal function in patients receiving immunotherapy for advanced cancer. Ann. Intern. Med.106(6), 817-822 (1987).

150 Dutcher J, Atkins MB, Margolin Ket al. Cytokine Working Group: Kidney cancer: the Cytokine Working Group experience (1986-2001): part II. Management of IL-2 toxicity and studies with other cytokines. Med. Oncol. 18(3), 209-219 (2001).

151 Locker GJ, Kapiotis S, Veitl M et al. Activation of endothelium by immunotherapy with interleukin-2 in patients with malignant disorders. Br. J. Haematol. 105(4), 912-919 (1999).

152 Yang JC, Sherry RM, Steinberg SM et al. Randomized study of high-dose and low-dose interleukin-2 in patients with metastatic renal cancer. J. Clin. Oncol. 21(16), 3127-3132 (2003).

153 McDermott DF, Regan MM, Clark JI et al. Randomized Phase III trial of high-dose interleukin-2 versus subcutaneous interleukin-2 and interferon in patients with metastatic renal cell carcinoma. J. Clin. Oncol. 23(1), 133-141 (2005).

154 Enk AH, Nashan D, Rübben A, Knop J. High dose inhalation interleukin-2 therapy for lung metastases in patients with malignant melanoma. Cancer 88(9), 2042-2046 (2000).

155 Hu P, Mizokami M, Ruoff G, Khawli LA, Epstein AL. Generation of low-toxicity interleukin-2 fusion proteins devoid of vasopermeability activity. Blood 101(12), 4853-4861 (2003).

156 Ribas A, Kirkwood JM, Atkins MB et al. Phase I/II open-label study of the biologic effects of the interleukin-2 immunocytokine EMD 273063 (hu14.18-IL2) in patients with metastatic malignant melanoma. J. Transl Med. 7, 68 (2009).

157 McDermott DF. Improving the therapeutic index of IL-2. Clin. Adv. Hematol. Oncol. 8(12), 862-864 (2010).

158 Bubeník J, Den Otter W, Huland E. Local cytokine therapy of cancer: interleukin-2, interferons and related cytokines. Cancer Immunol. Immunother. 49(2), 116-122 (2000). 
159 Parmiani G, Rivoltini L, Andreola G, Carrabba M. Cytokines in cancer therapy. Immunol. Lett. 74(1), 41-44 (2000).

160 Di Carlo E, Forni G, Lollini P, Colombo MP, Modesti A, Musiani P. The intriguing role of polymorphonuclear neutrophils in antitumor reactions. Blood 97(2), 339-345 (2001).

161 Ben-Efraim S. One hundred years of cancer immunotherapy: a critical appraisal. Tumour Biol. 20 (1), 1-24 (1999).

162 Epstein AL, Chen FM, Taylor CR. A novel method for the detection of necrotic lesions in human cancers. Cancer Res. 48(20), 5842-5848 (1988).

- Original report describing the use of tumor necrosis therapy antibodies to target necrotic regions of tumors in vivo.

163 Epstein AL, Chen D, Ansari A et al. Radioimmunodetection of necrotic lesions in human tumors using I-131 labeled TNT-1 $\mathrm{F}(\mathrm{ab}$ ') 2 monoclonal antibody. Antibody Immunoconjug. Radiopharm. 4, 151-161 (1991).
164 Hornick JL, Sharifi J, Khawli LA et al. A new chemically modified chimeric TNT-3 monoclonal antibody directed against DNA for the radioimmunotherapy of solid tumors. Cancer Biother. Radiopharm. 13(4), 255-268 (1998).

165 Li J, Hu P, Khawli LA, Epstein AL. LEC-chTNT-3 fusion protein for the immunotherapy of experimental solid tumors. J. Immunother. 26(4), 320-331 (2003).

166 Liu A, Hu P, Khawli LA, Epstein AL. B7.1/NHS76: a new costimulator fusion protein for the immunotherapy of solid tumors. J. Immunother. 29(4), 425-435 (2006).

167 Hornick JL, Khawli LA, Hu P, Sharifi J, Khanna C, Epstein AL. Pretreatment with a monoclonal antibody/interleukin-2 fusion protein directed against DNA enhances the delivery of therapeutic molecules to solid tumors. Clin. Cancer Res. 5(1), 51-60 (1995).
168 Chen S, Yu L, Jiang C et al. Pivotal study of iodine-131-labeled chimeric tumor necrosis treatment radioimmunotherapy in patients with advanced lung cancer. J. Clin. Oncol. 23(7), 1538-1547 (2005).

169 Yu L, Ju DW, Chen W et al. 131I-chTNT radioimmunotherapy of 43 patients with advanced lung cancer. Cancer Biother. Radiopharm. 21(1), 5-14 (2006).

170 Bose A, Taylor JL, Alber S et al. Sunitinib facilitates the activation and recruitment of therapeutic anti-tumor immunity in concert with specific vaccination. Int. J. Cancer. doi:10.1002/ijc.25863 (2010) (Epub ahead of print).

171 Lechner MG. Human Myeloid-Derived Suppressor Cells in Cancer: Induction, Functional Characterization, and Therapy. (Thesis Dissertation). University of Southern California: Systems Biology and Disease, CA, USA (2011).

172 Condamine T, Gabrilovich DI. Molecular mechanisms regulating myeloid-derived suppressor cell differentiation and function. Trends Immunol. 32(1), 19-25 (2011). 